\title{
Magnetospheric period oscillations at Saturn: Comparison of equatorial and high-latitude magnetic field periods with north and south Saturn kilometric radiation periods
}

\author{
D. J. Andrews, ${ }^{1}$ A. J. Coates, ${ }^{2,3}$ S. W. H. Cowley, ${ }^{1}$ M. K. Dougherty, ${ }^{4}$ L. Lamy, ${ }^{4,5}$ \\ G. Provan, ${ }^{1}$ and P. Zarka ${ }^{5}$ \\ Received 12 May 2010; revised 3 September 2010; accepted 9 September 2010; published 24 December 2010.
}

[1] It has recently been shown using Cassini radio data that Saturn kilometric radiation (SKR) emissions from the Northern and Southern hemispheres of Saturn are modulated at distinctly different periods, $\sim 10.6 \mathrm{~h}$ in the north and $\sim 10.8 \mathrm{~h}$ in the south, during the southern summer conditions that prevailed during the interval from 2004 to near-equinox in mid-2009. Here we examine Cassini magnetospheric magnetic field data over the same interval and show that two corresponding systems of magnetic field oscillations that have the same overall periods, as the corresponding SKR modulations, to within $\sim 0.01 \%$ are also present. Specifically, we show that the rotating quasi-dipolar field perturbations on southern open field lines and the rotating quasi-uniform field in the inner region of closed field lines have the same period as the southern SKR modulations, although with some intervals of slow long-term phase drift of unknown origin, while the rotating quasi-dipolar field perturbations on northern open field lines have the same period as the northern SKR modulations. We also show that while the equatorial quasi-uniform field and effective southern transverse dipole are directed down tail and toward dawn at southern SKR maxima, as found in previous studies, the corresponding northern transverse dipole is directed approximately opposite, pointing sunward and also slightly toward dawn at northern SKR maxima. We discuss these findings in terms of the presence of two independent high-latitude field-aligned current systems that rotate with different periods in the two hemispheres.

Citation: Andrews, D. J., A. J. Coates, S. W. H. Cowley, M. K. Dougherty, L. Lamy, G. Provan, and P. Zarka (2010), Magnetospheric period oscillations at Saturn: Comparison of equatorial and high-latitude magnetic field periods with north and south Saturn kilometric radiation periods, J. Geophys. Res., 115, A12252, doi:10.1029/2010JA015666.

\section{Introduction}

[2] Variations near the $\sim 11 \mathrm{~h}$ planetary rotation period in the power of Saturn's kilometric radiation (SKR) emissions, magnetospheric energetic ion and electron fluxes, and magnetospheric magnetic field were first detected during the planetary encounters by the Pioneer-11, Voyager-1, and Voyager-2 spacecraft [Kaiser et al., 1980; Desch and Kaiser, 1981; Warwick et al., 1981; Carbary and Krimigis, 1982; Espinosa and Dougherty, 2000; Espinosa et al., 2003a, 2003b]. More recent observations by the Cassini orbiter and the Hubble Space Telescope have shown that such

\footnotetext{
${ }^{1}$ Department of Physics and Astronomy, University of Leicester, Leicester, UK.

${ }^{2}$ Mullard Space Science Laboratory, University College London, Dorking, UK.

${ }^{3}$ Centre for Planetary Sciences, University College London/Birkbeck, London, UK.

${ }^{4}$ Blackett Laboratory, Imperial College London, London, UK.

${ }^{5}$ LESIA, Observatoire de Paris, CNRS, UPMC, Université Paris Diderot, Meudon, France.

Copyright 2010 by the American Geophysical Union. 0148-0227/10/2010JA015666
}

oscillations are also present in the location and power of Saturn's UV auroral emissions [Nichols et al., 2008, 2010; Provan et al., 2009b], the magnetopause and bow shock [Clarke et al., 2006, 2010a, 2010b], and magnetospheric cold and hot plasma populations, together with associated energetic neutral atom (ENA) emissions [Krupp et al., 2005; Clarke et al., 2006; Gurnett et al., 2007; Carbary et al., 2007a, 2007b, 2007c, 2008a, 2008b, 2009a, 2010; Arridge et al., 2008; Burch et al., 2009]. These studies have shown that the modulated SKR emissions are usually dominated by sources in the dawn-to-noon sector, such that their phase is to a first approximation independent of the position of the observer. However, the variations in the magnetospheric particles and field instead rotate around the planet with a similar period while also propagating radially outward such that the phase fronts form loosely wound spirals [e.g., Espinosa et al., 2003b; Cowley et al., 2006; Giampieri et al., 2006; Carbary et al., 2007b; Andrews et al., 2010; Clarke et al., 2010a].

[3] These oscillations were initially taken to be due to some anomaly (e.g., magnetic), undetected directly, that rotates rigidly with the planet [e.g., Galopeau et al., 1991]. 
(a) Quasi-uniform equatorial field Equatorial Plane

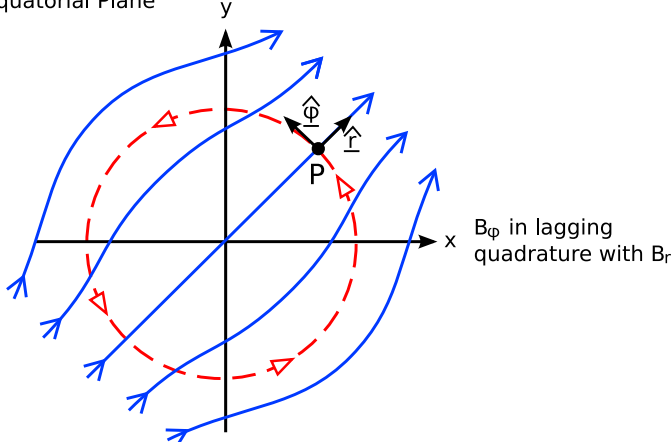

(b) Quasi-uniform equatorial field with apices to the north Meridional Plane

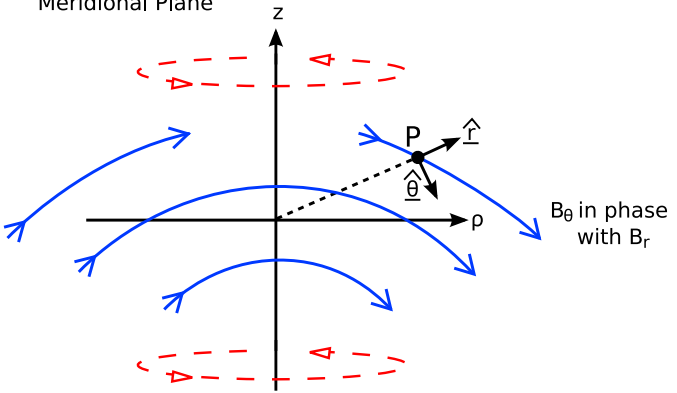

(c) Transverse quasi-dipolar field Equatorial plane

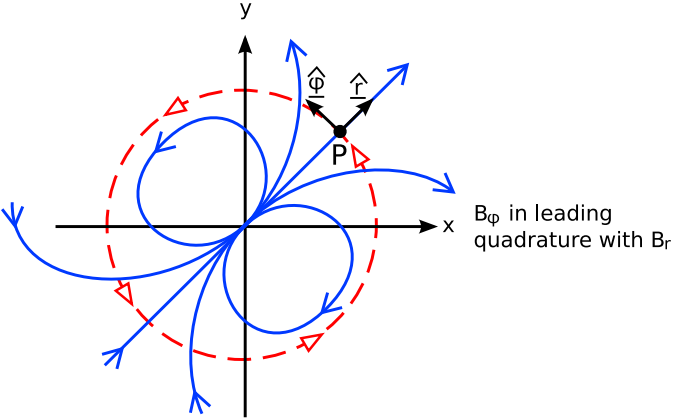

(d) Transverse quasi-dipolar field Meridional plane

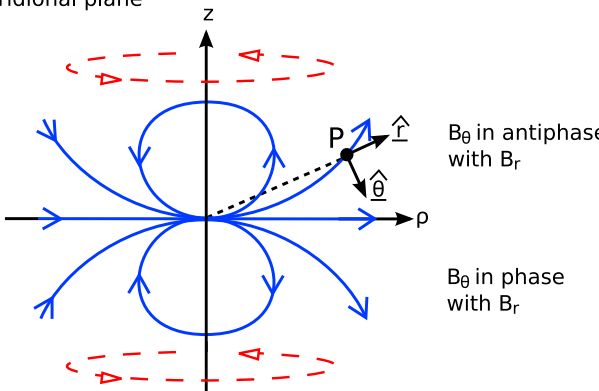

Figure 1. Sketches illustrating the polarization characteristics of the rotating perturbation field patterns discussed in this study, where Figures $1 \mathrm{a}$ and $1 \mathrm{~b}$ correspond to the rotating quasi-uniform field observed in the near-equatorial core region of the magnetosphere, while Figures $1 \mathrm{c}$ and $1 \mathrm{~d}$ correspond to the planet-centered rotating transverse dipole fields observed on high-latitude open field lines. (a and c) The equatorial $(x-y)$ plane in each case viewed from the north. (b and d) A meridional $(z-\rho)$ view, where $\rho$ lies in the direction in which the perturbation field points radially outward in the equatorial view. The arrowed blue lines in each panel indicate the perturbation field lines, while the arrowed red dashed lines indicate the sense of rotation of the field pattern with time. The phase relationships of the consequent oscillations in the field components at any spatial point in each panel (such as at the arbitrary points $\mathrm{P}$ shown) are indicated on the right.

Subsequent studies of the SKR modulations using Ulysses and Cassini data, however, have shown that the period varies by as much as $\sim 1 \%$ over yearly intervals [Galopeau and Lecacheux, 2000; Gurnett et al., 2005; Kurth et al., 2007, 2008], which is much too large a variation to be associated directly with the planet's rotation. Theoretical discussions have instead been related to systematic local time displacements of the SKR source associated with solar wind-magnetosphere interactions [Cecconi and Zarka, 2005] to centrifugally driven subcorotating convection systems initiated by internal plasma production [Gurnett et al., 2007; Goldreich and Farmer, 2007] and to seasonally related asymmetries involving the ring current and/or field-aligned current systems [Southwood and Kivelson, 2007; Khurana et al., 2009; Provan et al., 2009a].

[4] In this paper, we focus on these "magnetospheric period" oscillations in Saturn's magnetospheric magnetic field and their relation to the SKR modulations. Considering first the magnetic field oscillations, previous studies have shown that in the near-equatorial core region of the magnetosphere within radial distances of $\sim 10-15 \mathrm{R}_{\mathrm{S}}$, where the overall field is dominated by the quasi-dipolar field of the planet (Saturn's radius $R_{S}$ is $60268 \mathrm{~km}$ ), the oscillatory perturbation field takes the form of a quasi-uniform field in the equatorial plane that rotates in the sense of planetary rotation with a period close to the SKR period [Espinosa et al., 2003a, 2003b; Southwood and Kivelson, 2007; Andrews et al., 2008, 2010; Provan et al., 2009a]. The polarization characteristics of such a rotating perturbation field are illustrated for future reference in Figure 1a, which shows a view of the equatorial $(x-y)$ plane looking from the north. The quasi-uniform perturbation field is indicated schematically by the blue lines, which is such that a maximum in the spherical polar radial $(r)$ field component occurs at point $\mathrm{P}$ at the instant depicted. Spherical polar field components expressed with respect to the northern rotation and magnetic $(z)$ axis are employed throughout this paper. As the perturbation field pattern rotates around the axis as indicated by the arrowed red dashed circle, it can be seen that the maximum in the $r$ component at $\mathrm{P}$ will be followed a quarter cycle later by a maximum in the azimuthal $(\varphi)$ component. For such a field therefore the $\varphi$ component oscillates in lagging quadrature with the $r$ component, as indicated by the above-cited authors. Oscillations in the colatitudinal $(\theta)$ field component are also present in the equatorial region, which are in phase with the $r$ component [Andrews et al., 2008, 2010; Provan et al., 2009a]. The nearequatorial perturbation field lines thus form arches with apices in the north, as illustrated schematically in Figure 1b, 
which shows perturbation field lines projected onto the principal magnetic meridian $(z-\rho)$ of the disturbance (where $\rho=\sqrt{x^{2}+y^{2}}$ lies in the direction in which the perturbation field points radially outward in the equatorial view). The phase of the field oscillations relative to the SKR modulations is found to be such that the SKR power peaks when the quasiuniform field points radially outward in the postmidnight sector, at $\sim 02 \mathrm{~h}$ local time (LT). However, considerable "jitter" in the phase of the equatorial magnetic oscillations is also found to be present, with amplitude $\sim 20^{\circ}$ [Andrews et al., 2008]. We note in this context that variations in the SKR phase on 20-30 day time scales have also been found to be present, which appear to be connected to variations in the solar wind speed [Zarka et al., 2007].

[5] Magnetic oscillations in the high-latitude magnetosphere on open field lines were first studied by Provan et al. [2009a], who found that, unlike the equatorial region, the polarization characteristics are those of a planet-centered rotating transverse dipole in both hemispheres. The characteristics of such a rotating field are illustrated schematically in Figures 1c and 1d, which have similar formats to Figures $1 \mathrm{a}$ and $1 \mathrm{~b}$. Consideration of the equatorial projection in Figure 1c shows that in this case the $\varphi$ component oscillates in leading quadrature with the $r$ component (both on and away from the equator), while Figure 1d shows that the $\theta$ and $r$ components are in-phase south of the equatorial plane (as in the core region) but are in antiphase north of the equatorial plane. These are the relative phases between the field components found on open field lines in both hemispheres by Provan et al. [2009a]. These authors also showed that the $r$ and $\theta$ oscillations at southern high latitudes are approximately in-phase with those within the core region, such that the $\varphi$ components in the two regions oscillate approximately in antiphase. Thus, the effective transverse magnetic dipole moment vector corresponding to the Southern Hemisphere oscillations, which rotates in-phase with the $r$ component of the perturbation field (see Figure 1c), is directed approximately parallel to the quasi-uniform field in the core region. This effective transverse dipole thus again points down tail and toward dawn at SKR maxima. However, Provan et al. [2009a] also found that the relative phase between the core region oscillations and the northern high-latitude quasi-dipolar oscillations is somewhat scattered, such that the relative orientation of the effective transverse dipoles in the two hemispheres remains uncertain.

[6] Considering now the SKR emissions, these are believed to be generated predominantly in the extraordinary $(\mathrm{X})$ mode by accelerated auroral electrons on highlatitude field lines via the cyclotron maser instability, with emissions generated in the Northern and Southern hemispheres having opposite circular polarization $[W u$ and Lee, 1979; Warwick et al., 1981; Lecacheux and Genova, 1983; Galopeau et al., 1995; Zarka, 1998; Lamy et al., 2008a, 2008b, 2010; Cecconi et al., 2009]. Emission sources may occur at any local time, colocated with the UV auroral oval [Lamy et al., 2009], but as indicated above are generally dominated by those in the dawn-to-noon sector. Most recently, however, it has been discovered that in the initial Cassini era (2004-2008), emissions from the two hemispheres are modulated at distinctly different periods, $\sim 10.6 \mathrm{~h}$ in the north and $\sim 10.8 \mathrm{~h}$ in the south [Gurnett et al., 2009a], while the overall emitted power during the interval is generally dominated by the longer-period emissions from the Southern Summer Hemisphere. The presence of two distinct SKR modulation periods was previously reported in Cassini data by Kurth et al. [2008] and in Voyager era data by Desch and Kaiser [1981] (with closer periods in that case of 10.66 and $10.69 \mathrm{~h}$ ). Corresponding distinct modulation periods have also subsequently been reported in both auroral hiss and magnetospheric energetic electron data [Gurnett et al., 2009b; Carbary et al., 2009b].

[7] From the magnetic field results presented by Andrews et al. [2008] and Provan et al. [2009a], it is evident that the magnetic field oscillations observed in the near-equatorial and southern high-latitude regions in the initial Cassini era relate to the dominant longer-period southern SKR modulations. Thus, the phase relationships outlined above between the field oscillations in these regions and the SKR power modulations refer specifically to radio emissions originating in the Southern Hemisphere. The relationships between the field oscillations and the shorter-period SKR modulations originating from the Northern Hemisphere remain to be investigated in detail. However, at the magnetospheres of outer planets (MOP) meeting held in Köln, Germany, in July 2009, A. Lecacheux and D.J. Southwood provided initial evidence that the quasi-dipolar northern high-latitude field oscillations occur at the shorter northern SKR period (see "Studies of high-latitude phase pulses in the Saturnian SKR radio and comparison with Cassini magnetic field data," MOP Abstracts 2009, p. 17), thus potentially providing a basis for understanding the more scattered northern phase results of Provan et al. [2009a].

[8] In the previous study by Provan et al. [2009a], the phases of the near-equatorial and northern and southern high-latitude magnetic field oscillations were thus (in effect) compared with those of the dominant southern SKR modulations over the interval from Cassini Saturn orbit insertion (SOI) in mid-2004 to the end of 2007. In this paper, we now considerably extend this analysis to cover all such magnetic field data obtained in the preequinox interval to mid-2009 (Saturn's vernal equinox occurred on 11 August 2009) and compare the phases of the field oscillations with both the northern and southern SKR modulations. We show that the periods of the equatorial and southern high-latitude oscillations are close to the southern SKR period throughout this interval, while the period of the northern high-latitude oscillations is close to the northern SKR period.

\section{Data Selection, Processing, and Analysis}

\subsection{Magnetic Oscillation Model}

[9] The basis of the analysis presented here follows that previously employed by Andrews et al. [2008] and Provan et al. [2009a], where the phase of the magnetic field oscillations is obtained (to modulo $360^{\circ}$ ) relative to those of the SKR modulations, employed as exact "guide phases." Assuming that the magnetic oscillations rotate in the sense of planetary rotation as a " $m=1$ " disturbance (i.e., varying with azimuth $\varphi$ as $e^{-j m \varphi}$ with $m=1$ ), as opposed to the modulations of the SKR emissions whose phase is 


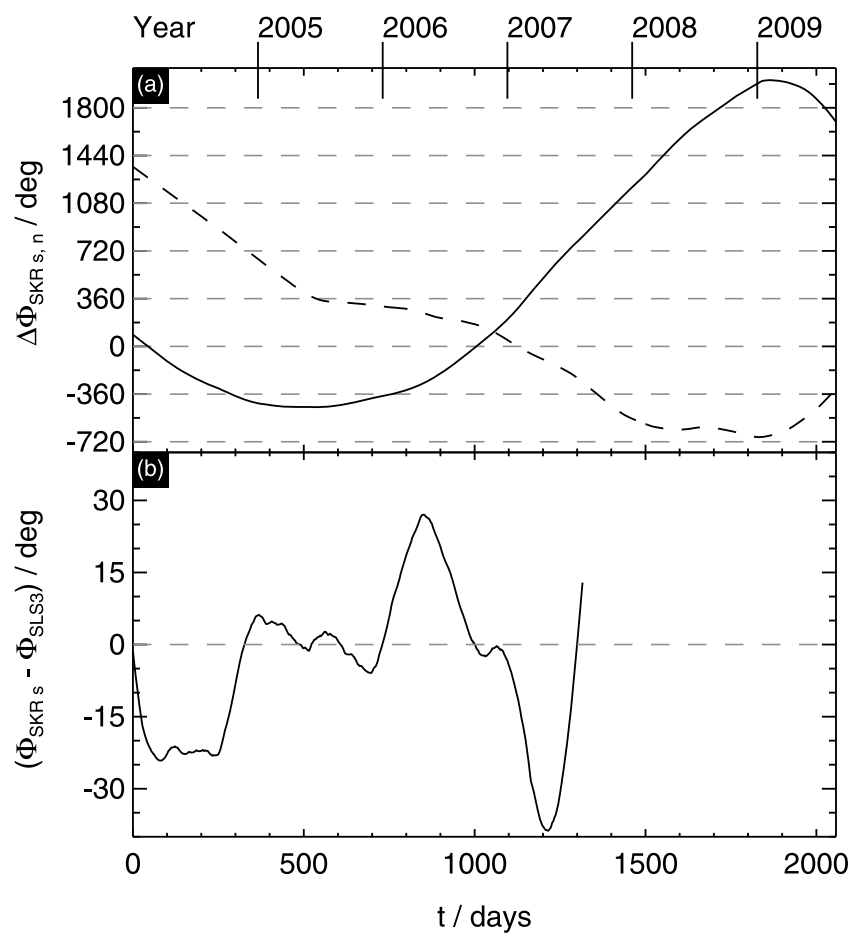

Figure 2. Plots relating to the numerical Northern and Southern hemisphere SKR phases employed in this paper given by equation (2a), shown over the interval $t=0-2057$ days, where $t=0$ corresponds to the start of 1 January 2004 . (a) The empirically determined phase of the northern (dashed line) and southern (solid line) SKR modulations relative to oscillations with fixed periods of exactly 10.6 and $10.8 \mathrm{~h}$, respectively, as given by equation (2b). (b) The difference between the numerical Southern Hemisphere SKR phase employed in this paper, $\Phi_{\mathrm{SKR}}$ s , and the polynomial-based SLS3 SKR phase model of Kurth et al. [2008], $\Phi_{\mathrm{SLS} 3}$, shown versus time $t$ over the interval of validity of the SLS3 phase, $t=0-1317$ days. Calendar year boundaries are shown at the top of the plot.

independent of position, we express the variation of each field component $B_{i}$ as

$$
B_{i}(\varphi, t)=B_{i 0} \cos \left(\Phi_{\mathrm{SKR} \mathrm{n}, \mathrm{s}}(t)-\varphi-\psi_{\mathrm{Min}, \mathrm{s}}\right) .
$$

In this expression $\varphi$ is the azimuthal angle measured positive from noon toward dusk, $\Phi_{\mathrm{SKR} \mathrm{n,s}}(t)$ is the phase of the northern ("n") or southern ("s") SKR modulations defined such that emission maxima occur when $\Phi_{\mathrm{SKR} \mathrm{n,s}}(t)=360 \mathrm{~N}$ degrees for integer $N$, and $\psi_{\mathrm{Min}, \mathrm{s}}$ is the corresponding relative phase of the oscillation in magnetic field component $i$. The latter phase is determined separately for equatorial and high-latitude field data from individual spacecraft orbit revolutions ("Revs") by fitting equation (1) to magnetic field data suitably processed as described below, with $\varphi$ given by the azimuthal angle of the spacecraft (equivalent to LT), and $B_{i 0}$ and $\psi_{\mathrm{Min}, \mathrm{s}}$ as free parameters. If the period of the rotating magnetic oscillations is the same as that of the corresponding SKR modulations, the $\psi_{\mathrm{Mi}}$ values obtained will be constant from pass to pass for each field component, with the phase differences between the components depending on the oscillation polarization characteristics as described in section 1 (Figure 1). Deviations between the magnetic and SKR periods are then revealed by changes in the $\psi_{\mathrm{Mi}}$ values for each component from Rev to Rev.

\subsection{Northern and Southern SKR Phases}

[10] In this study, we employ the phases of the SKR modulations in each hemisphere, $\Phi_{\mathrm{SKR} \text { n,s }}(t)$, derived from Cassini RPWS instrument data [Gurnett et al., 2004] by L. Lamy, P. M. Zarka, and B. Cecconi ("SKR variable periods: investigation of north/south fluctuations from short to longterm time scales," Abstract SM41A-1692, Fall Meeting, American Geophysical Union, San Francisco, USA, 2009, manuscript in preparation). The numerical phase data set, along with a summary of its derivation, is available online at the LESIA Observatoire de Paris Web site (http://www. lesia.obspm.fr/kronos/guest.php). Briefly, the radio data are first separated into northern and southern emissions on the basis of their polarization and the latitude of the spacecraft, with right- and left-handed emissions corresponding to the Northern and Southern hemispheres, respectively. The separated flux densities are then integrated in the SKR frequency band $40-500 \mathrm{kHz}$ and normalized with respect to radial distance from the planet, and the dominant period in each hemisphere $\tau_{\text {SKR n,s }}(t)$ is then computed using a LombScargle analysis with a 200 day sliding window [Lomb, 1976; Scargle, 1982; Horne and Baliunas, 1986]. This time series is then numerically integrated to determine the SKR phase for each hemisphere using

$$
\Phi_{\mathrm{SKR} n, \mathrm{~s}}(t)=360 \int_{0}^{t} \frac{\mathrm{d} t}{\tau_{\mathrm{SKR} \mathrm{n}, \mathrm{s}}}+\Phi_{0 \mathrm{n}, \mathrm{s}},
$$

where $t=0$ corresponds to the start of day 1 of 2004 and $\Phi_{0 \mathrm{n}, \mathrm{s}}$ is a constant determined (to modulo $360^{\circ}$ ) by requiring SKR power maxima to correspond to phases of $360 \mathrm{~N}$ degrees as indicated above.

[11] The northern and southern SKR phases so determined are shown in Figure 2a over the interval $t=0-2057$ days (from start of 1 January 2004 to end of 19 August 2009), encompassing the interval for which magnetic field phases are determined in this paper as will be outlined in section 2.3 below. The northern phase (dashed line) is shown relative to the phase of a fixed period of exactly $10.6 \mathrm{~h}$, while the southern phase (solid line) is shown relative to that of a fixed period of exactly $10.8 \mathrm{~h}$. Specifically, the quantities shown are

$$
\Delta \Phi_{\mathrm{SKR}, \mathrm{s}}(t)=\left(360^{\circ} t / \tau_{\mathrm{n}, \mathrm{s}}^{*}\right)-\Phi_{\mathrm{SKRn}, \mathrm{s}}(t),
$$

where the phases are expressed in degrees, $\tau_{\mathrm{n}}^{*} \equiv 10.6 \mathrm{~h}$, and $\tau_{\mathrm{s}}^{*} \equiv 10.8 \mathrm{~h}$. Intervals for which these quantities are approximately constant thus indicate an SKR modulation period that is close to that of the respective reference period, while positive or negative gradients indicate a period that is longer or shorter, respectively. The overall deviation about near-linear behavior of these phases can be seen to be by around a cycle or two in either direction over this $\sim 5.6$ year interval. 
[12] We also note that the previous studies of the phases of the rotating magnetic oscillations by Andrews et al. [2008] and Provan et al. [2009a] employed the SKR phase models provided by Kurth et al. [2007, 2008], in which the phase is expressed as a polynomial in time $t$ obtained from fits to the SKR phase data. The most recent model of Kurth et al. [2008] employed by Provan et al. [2009a] is expressed as a fifth-order polynomial, obtained over the interval $t=0-1317$ days (to 10 August 2007). To gauge the changes in the results expected over this interval due to differences between the SKR phase models, in Figure $2 \mathrm{~b}$ we plot the difference between the Southern Hemisphere numerical SKR phase employed here and the corresponding value of the Kurth et al. [2008] (SLS3) model, $\Phi_{\text {SKR s }}-\Phi_{\text {SLS3 }}$ over the interval of validity of the latter. With the exception of the region near the end of the end of the interval, it can be seen that deviations are generally limited to within about $\pm 20^{\circ}$, the overall RMS value of the difference being $16^{\circ}$. Such differences are thus much less significant than the overall phase deviations due to the longer-term variability of the SKR modulation period indicated in Figure 2a. As will be seen in section 3 below, these differences are only of minor significance to the magnetic field phase results presented here, such that our results may be compared directly with those of Andrews et al. [2008] and Provan et al. [2009a] despite the differing SKR phases employed.

\subsection{Selection and Processing of Magnetic Field Data}

[13] We now discuss the selection methods and processing procedures employed for the magnetic field data. Intervals of core and high-latitude field data to be employed in the analysis have first been identified using an automated procedure that has been checked and moderated (if required) by visual inspection of the data. As discussed further below, core intervals in this study correspond to regions with dipole $L \leq 8$, while the high-latitude data correspond to northern and southern open field regions within radial distances of 25 $\mathrm{R}_{\mathrm{S}}$ where warm and hot electron fluxes are close to instrumental background (see below). We note in passing that these radial distance limitations have resulted in our taking no account in equation (1) of changes in magnetic oscillation phase with radius $r$. Detailed examination has shown that radial phase gradients within the core of $\sim 2 \operatorname{deg} R_{S}^{-1}$ [Andrews et al., 2010] produce insignificant effects on our results, while variability in the phase determinations has not allowed us to verify the presence or otherwise of gradients of similar order in the high-latitude data employed here.

[14] Region identification using the above criteria is illustrated in Figure 3 for Cassini Rev 95 in December 2008, on which, somewhat unusually, the spacecraft trajectory allowed sampling of all three regions considered. On this Rev, the spacecraft passed from a near-equatorial apoapsis at a radial distance of $\sim 20.3 \mathrm{R}_{\mathrm{S}}$ in the prenoon sector (10.6 h LT) near the beginning of the plot, over the north polar region to a near-equatorial periapsis at a radial distance of $\sim 5.2 \mathrm{R}_{\mathrm{S}}$ in the premidnight sector $(22.6 \mathrm{~h} \mathrm{LT})$ near the center of the plot, and then over the southern polar region to apoapsis again toward the end of the plot. From top to bottom of Figure 3, we show an electron spectrogram between $0.6 \mathrm{eV}$ and $28 \mathrm{keV}$ from the CAPS-ELS instrument [Young et al., 2004], followed by residual and filtered spherical polar $(r, \theta, \varphi)$ magnetic field components obtained by the fluxgate magnetometer [Dougherty et al., 2004]. The residual field data have the "Cassini SOI" internal model subtracted [Dougherty et al., 2005], but since this field is exactly symmetric about the planet's spin axis with zero azimuthal component, the azimuthal field shown is that measured. As in previously related analyses, the residual field data have then been band-pass filtered between periods of 5 and $20 \mathrm{~h}$ using a standard Lanczos filter in order to isolate oscillations centered in the magnetospheric period band (see Andrews et al. [2008] and Provan et al. [2009a] for further discussion).

[15] Northern and southern open field regions, designated " $\mathrm{O}_{\mathrm{n}}$ " and " $\mathrm{O}_{\mathrm{s}}$ " in Figure 3, are identified specifically by requiring the integrated electron counts between 50 and 500 $\mathrm{eV}$ to be close to instrument background (noting that the variable intense fluxes below a few tens of $\mathrm{eV}$ in Figure 3 are spacecraft photoelectrons), with such intervals persisting for at least $\sim 6 \mathrm{~h}$ being selected for study. Short data segments with moderately higher electron count rates are allowed within such intervals, provided they are not recurrent near the magnetospheric period, potentially indicative of an oscillating boundary region. In Figure 3, both northern and southern open field regions so identified, bounded by the vertical dashed lines, have durations of $\sim 2$ days. Since this corresponds to about four oscillations at either northern or southern SKR periods, the oscillation phases $\psi_{\mathrm{Mi}}$ in each field component can be well determined by least squares fitting equation (1) to the filtered residual data. Uncertainties in the fitted phase are estimated by determining the phase shifts required to give a significant $10 \%$ increase in the root mean square (RMS) deviation between the field data and the fitted model.

[16] We note, however, that on other Revs during the interval of study, regions of open field data can be observed closer to the planet during which large and rapid field variations also occur due to spacecraft motion through the high-latitude field perturbations produced by the nearequatorial ring current and magnetotail. Such variations then introduce significant power into the filter band that is unrelated to the magnetospheric period oscillations, so that the phases determined from such data must be excluded from the analysis. However, since the ring current fields are observed to be near-axisymmetric to a first approximation with little azimuthal component [e.g., Bunce et al., 2007], related to the major current contribution of the near-axisymmetric cool water ion plasma transported outward from the Enceladus torus [Kellett et al., 2010; Sergis et al., 2010], this problem affects only the $r$ and $\theta$ components of the field, while phases can still be determined from the $\varphi$ component.

[17] Turning now to the data from the closed field core region, designated "C" in Figure 3, we note that the limitation of the region to dipole $L \leq 8$ employed here is more restrictive than in the studies by Andrews et al. [2008] and Provan et al. [2009a], where dipole $L \leq 12$ was employed. This limitation is required to exclude the highly structured magnetic signatures of field-aligned currents that flow predominantly in the outer magnetosphere between the core and the open field region [Talboys et al., 2009a, 2009b] at lower latitudes on the nightside than on the dayside. In Figure 3, it can be seen that these current signatures are 
localized mainly in the short intervals between the regions " $\mathrm{C}$ " and " $\mathrm{O}_{\mathrm{n}, \mathrm{s}}$ " on either side of periapsis. On more extended lower-latitude traversals through the core region during some intervals of the Cassini mission, the core oscillation phases can be determined for all three field components by fitting equation (1) to the filtered data, as for the high-latitude data. However, ring current fields can again introduce spurious phases into the $r$ and $\theta$ component data on some Revs, which also must be rejected, as before leaving data only for the $\varphi$ component. This restriction on the $r$ and $\theta$ component data also applies to shorter highly inclined passes through the core region, such as that shown

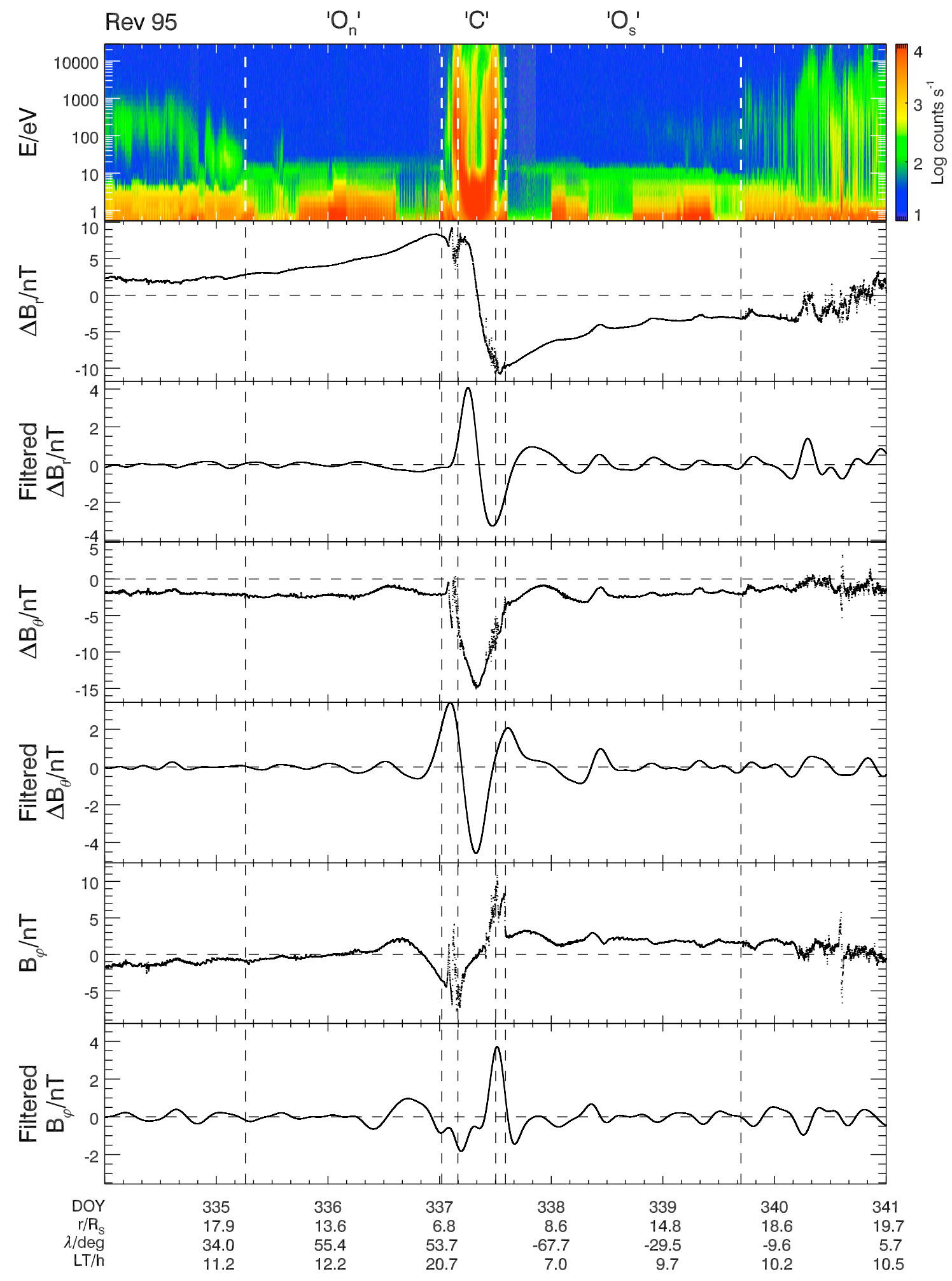

Figure 3 


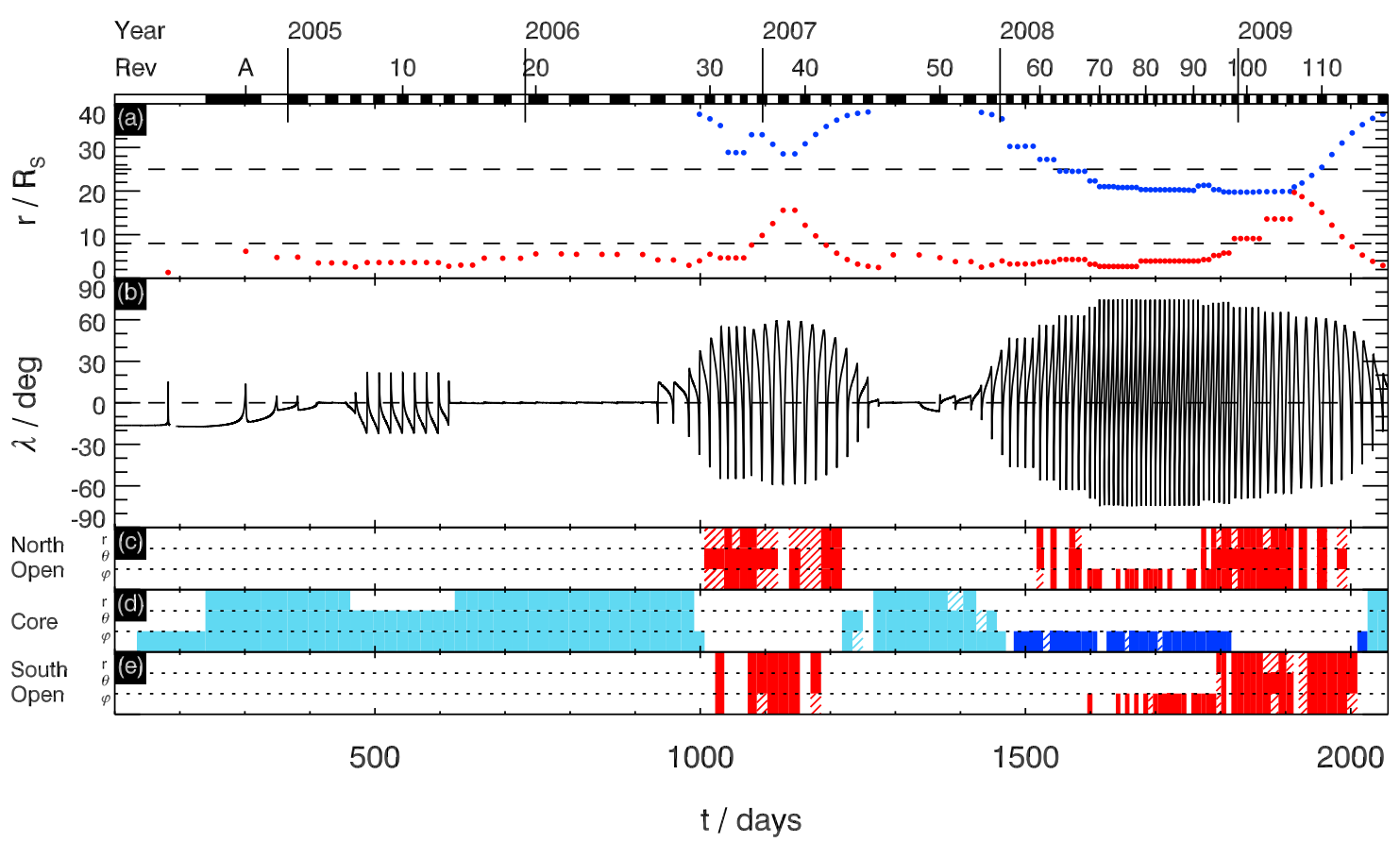

Figure 4. Plot showing characteristics of the Cassini orbital tour that govern measurements of the magnetic field phase in each of the three regions studied in this paper. The interval covered by the plot is from $t=100-2057$ days, spanning mid-April 2004 to mid-August 2009. Alternate black and white boxes at the top of the plot indicate the duration of each spacecraft Rev from apoapsis to apoapsis spanning SOI to Rev 116, marked and labeled at the periapsis of every tenth Rev. Year boundaries are also marked. (a) The radial distance $\left(\mathrm{R}_{\mathrm{S}}\right)$ of periapsis (red points) and apoapsis (blue points) on each Rev, while the horizontal dashed lines shown at 8 and $25 \mathrm{R}_{\mathrm{S}}$ indicate the equatorial extent of the core region (dipole $L \leq 8$ ) and the outer radius of the open field regions employed in this study, respectively. (b) The latitude $\lambda$ of the spacecraft (degrees), showing the two extended high-latitude intervals of the orbital tour. (c-e) The Revs that yield magnetic phase data for the northern open region, the core region, and the southern open region, respectively. Each of these panels is subdivided into three, corresponding to the three spherical polar field components as marked. A particular Rev is shown colored, red for the two open regions and blue for the core region, if a phase measurement for that region and field component was obtained from that Rev. In the core region, light blue indicates phase data determined from filtered magnetic field data, while dark blue indicates phase data determined from unfiltered azimuthal component data during short traversals of the core region, as outlined in section 2. Blank areas indicate that valid phase data were not obtained for a particular region and component for a particular Rev. Hatching of an appropriate color indicates phase data that have further been excluded due to limitations on the RMS deviation between model and data and on the oscillation amplitude, as discussed in section 2 .

in Figure 3, where, as can be seen, the filtered $\varphi$ component data are also contaminated by the field-aligned current signatures on either side. In these cases, therefore, we fit equation (1) to the unfiltered core region $\varphi$ component data (only). This should in principle still give reasonable phase values even for relatively short data segments (e.g., $\sim 8 \mathrm{~h}$ in Figure 3) since there are no other major sources of azimuthal field within the core. Even so, the phases so determined are clearly expected to have greater uncertainty (again determined using the above algorithm) and scatter than those determined from filtered data acquired over several oscillations.

[18] We finally note that phase data are also excluded from our analysis when the RMS deviation between the data

Figure 3. Plot showing 7 days of electron flux and magnetic field data from Cassini Rev 95 during December 2008 (days 334-340 inclusive), typical of the orbit geometry during this phase of the mission. The top shows an electron energy-time spectrogram covering the energy range $0.6 \mathrm{eV}$ to $28 \mathrm{keV}$, while the lower three pairs show residual and band-pass filtered magnetic field data for each of the three spherical polar field components referenced to the planet's spin and magnetic axis. The residual components have the Cassini SOI internal field model subtracted, whereas because the azimuthal field of this model is zero, this field component is shown as measured. Spacecraft position information is indicated at the bottom, specifically the radial distance $\left(\mathrm{R}_{\mathrm{s}}\right)$, the latitude (degrees), and the local time (hours). The three intervals whose data have been selected for oscillation phase determinations on this Rev are delimited by the vertical dashed lines and are labeled " $\mathrm{O}_{\mathrm{n}}$ " " $\mathrm{O}_{\mathrm{s}}$," and " $\mathrm{C}$ " for the Northern Hemisphere and Southern Hemisphere open field regions and the closed field core region, respectively. 
and equation (1) becomes sufficiently large or when the fitted amplitude becomes sufficiently small. Reasonable empirically determined limits are RMS deviations more than twice the fitted amplitude and field oscillation amplitudes less than $0.2 \mathrm{nT}$. In practice, these limits result in the removal of only $\sim 14 \%$ of the otherwise acceptable phase values determined here.

\subsection{Overview of Magnetic Oscillation Phase Data Availability}

[19] In Figure 4, we provide an overview of those Cassini Revs, governed principally by the orbit characteristics, which yield magnetic phase data for each of the three regions examined here (i.e., the core region and the northern and southern open field regions), within the limitations outlined above. The plot covers the interval from $t=100$ (beginning of 10 April 2004) to $t=2057$ days (end of 19 August 2009, just past the vernal equinox), thus spanning the whole of the Cassini orbital tour from SOI in mid-2004 to Rev 116. The intervals of individual Revs defined from apoapsis to apoapsis are indicated by the successive black and white stripes at the top of Figure 4, which are marked and numbered at the periapsis of every tenth Rev. Year boundaries are also marked. Data from Figures $4 \mathrm{a}$ and $4 \mathrm{~b}$ beneath this then show some relevant characteristics of the spacecraft orbit. Figure $4 \mathrm{a}$ shows the radial distance of apoapsis and periapsis (blue and red dots, respectively), while the horizontal dashed lines at 8 and $25 \mathrm{R}_{\mathrm{S}}$ indicate the equatorial extent of the core region and the outer radius of the open field regions employed here, respectively. Beneath this in Figure $4 \mathrm{~b}$ we plot the latitude $\lambda$ of the spacecraft, showing the presence of two intervals of high-latitude orbits that allow observations of the northern and southern open field regions. We also note the increases in periapsis radius during parts of these intervals that result in a lack of simultaneous core observations during portions of both of them.

[20] The consequent availability of magnetic phase data for each of the three regions is then shown on a Rev-by-Rev basis in Figures $4 c-4 e$ at the bottom, where Figure $4 c$ corresponds to the northern open field region, Figure $4 \mathrm{~d}$ corresponds to the core region, and Figure $4 \mathrm{e}$ corresponds to the southern open field region. Figures $4 \mathrm{c}-4 \mathrm{e}$ are each subdivided into three regions corresponding to the three spherical polar field components, as marked and colored red in the open field regions and blue in the core region when a phase value for a specific field component has been determined for a particular Rev. Light blue coloring in the core region corresponds to Revs for which phase values have been determined using filtered data, while dark blue coloring corresponds to Revs for which core phase determinations have been made using shorter intervals of unfiltered $\varphi$ component data only. Blank intervals then indicate a lack of corresponding data because either the spacecraft did not enter a particular region on a particular Rev, or the phase value for a particular component is invalid for reasons given above, or there is an extended data gap such that phase values cannot be determined. Phase values discounted due to limitations on the RMS deviation and oscillation amplitude are shown hatched in the appropriate color.

[21] Figure 4 thus provides a summary of the extent and limitations of the magnetic field data set employed here, together with its relationship to previous studies. We note that the first interval of core region data, obtained from the near-equatorial orbits of Rev A to Rev 28 (into the second half of 2006), was that studied by Andrews et al. [2008], while Provan et al. [2009a] extended this study to include data from both SOI and the second near-equatorial mission interval, to Rev 54 at the end of 2008. Subsequent core data, new to this study, span 2008 to mid-2009, and predominantly consist of measurements obtained from unfiltered $\varphi$ component data as illustrated in Figure 3 (the last two Revs excepted). Similarly, the first interval of open field data, obtained between August 2006 and April 2007 on Revs 30-43, was that studied by Provan et al. [2009a]. The second longer interval of open field data between February 2008 and June 2009, corresponding to Revs 60-113, is newly studied here. We note that the latter interval contains the only extended period of essentially simultaneous core and open field phase data presently available.

\section{Magnetic Oscillation Phase Results}

[22] We now present the magnetic oscillation phase data determined for each of the three regions as outlined in section 2. The results of Provan et al. [2009a] indicate that the oscillations on Southern Hemisphere open field lines during the interval they investigated have a period close to the southern SKR period, as may be expected, while the results of both Andrews et al. [2008] and Provan et al. [2009a] show that the oscillations in the core region have the same southern SKR period. The results obtained by Provan et al. [2009a] for the Northern Hemisphere open field line region with respect to the southern SKR period were more scattered. In this section, we thus begin by reexamining the issue of the phase and period of the oscillations in the southern open region and core region over the more extended interval examined here and then consider the oscillations in the northern open region.

\subsection{Southern Hemisphere Open Field Lines}

[23] Results for the southern open field region are shown in Figure 5, where the plot now spans the two high-latitude intervals of the Cassini mission between August 2006 and August 2009 rather than the whole mission interval as shown in Figure 4. Specifically we show the interval from $t=950$ to 2057 days, corresponding to Revs 27-116, as indicated by the bar at the top of Figure 5, which has the same format as in Figure 4. Figure 5a shows the magnetic oscillation phases $\psi_{\text {Mi s }}$ relative to the Southern Hemisphere SKR phase determined using equation (1) for each field component as available. Noting that these phases are determined only to modulo $360^{\circ}$, we have plotted these data over two cycles between $-180^{\circ}$ and $+540^{\circ}$, with each data point thus being shown twice, in order to demonstrate the banded nature of the values, if such is present. The data obtained from the $r, \theta$, and $\varphi$ components of the field are indicated by the red, green, and blue symbols, respectively, with vertical bars showing their uncertainties determined as described in section 2. As indicated in section 1, the previous results of Provan et al. [2009a] have shown that the polarization of the oscillations on southern open field lines corresponds to a planet-centered rotating transverse dipole, with $r$ and $\theta$ oscillations in-phase, and $\varphi$ in leading quad- 


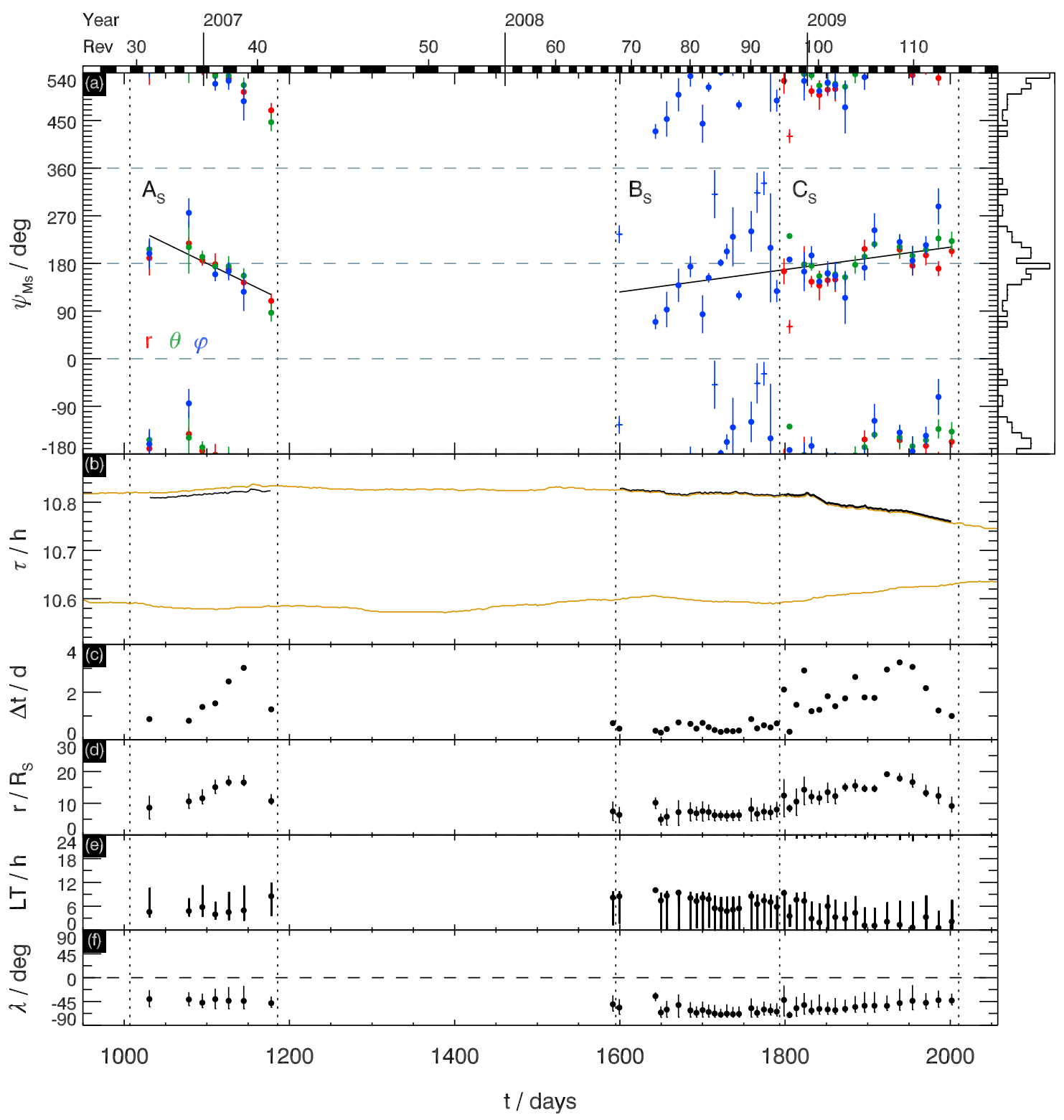

Figure 5. Plot showing results for the Southern Hemisphere region of open field lines, spanning the interval from $t=950-2057$ days, corresponding to Revs 27-116 as shown by the bar at the top of the plot having the same format as in Figure 4. (a) The magnetic oscillation phase values relative to the southern SKR modulation phase, plotted over two cycles between $-180^{\circ}$ and $+540^{\circ}$ so that each data point is plotted just twice. Phase data for the $r, \theta$, and $\varphi$ components are shown by the red, green, and blue symbols, respectively, with vertical bars indicating the uncertainty in each measurement. The $r$ and $\theta$ component phases are shown as measured, whereas because the $\varphi$ component oscillations are in leading quadrature, $90^{\circ}$ has been added to these values to bring them to a common phase with the $r$ and $\theta$ component data. Piecewise linear fits to these phase data are shown by the black solid lines. Phase data included in these fits are shown by the colored dots, while values excluded are shown by crosses (no dots), as outlined in the text. Banding of the overall phase values is indicated in the histogram plotted vertically to the right of Figure 5a, computed in $10^{\circ}$ phase bins. (b) Solid yellow lines show the southern (upper, longer) and northern (lower, shorter) hemisphere SKR periods, in hours, while the black lines show the magnetic field oscillation periods corresponding to the linear fits shown by the black solid lines to the phase data in Figure 5a. (c) The duration (days) of the southern open field intervals employed to determine the magnetic field phases. Also shown are corresponding spacecraft position data, specifically (d) the radial distance $\left(R_{S}\right)$, (e) local time (hours), and (f) latitude (deg). Black circles in Figures 5d-5f indicate the spacecraft position at the center of each interval, while the vertical bars indicate the range of the relevant quantity over the whole interval employed in the determination of the magnetic field phases. 
rature with both (Figures 1c and 1d). In anticipation of this result, while the $r$ and $\theta$ component phases in Figure 5 are shown as measured, we have added $90^{\circ}$ to the $\varphi$ component phases to bring them to a common value with the $r$ and $\theta$ component phases, if they are indeed in leading quadrature. It can be seen that the phases so plotted have common values for all field components throughout the interval when simultaneous data are available, thus confirming the polarization found by Provan et al. [2009a]. The yellow lines in Figure $5 \mathrm{~b}$ then show the southern (upper) and northern (lower) hemisphere SKR modulation periods (hours) determined as outlined in section 2, while the black lines show inferred magnetic oscillation periods as will be discussed below. Figures $5 c-5 f$ then provide information about the southern open field intervals used to derive the phase data. Figure $5 \mathrm{c}$ indicates the duration $\Delta t$ in days of each interval for which magnetic field phases are determined, while Figures $5 \mathrm{~d}-5 \mathrm{f}$ indicate the spacecraft location during each interval, with regard to radial distance $\left(\mathrm{R}_{\mathrm{S}}\right)$, LT (hours), and latitude (degrees), respectively. In the latter panels, the black dots correspond to the center of each open field interval, while the vertical bars indicate the range of the parameter over the open field interval.

[24] The centrally important result in Figure 5 is the clear banding of the magnetic phase values about $\sim 180^{\circ}$ (modulo $360^{\circ}$ ) over the whole interval of the plot, spanning $\sim 2.5$ years. This implies that the magnetic field oscillations on southern open field lines are well organized by and thus have a period very close to that of the Southern Hemisphere SKR modulations. The banding is quantified by the histogram of phase values (including all field components) plotted vertically on the right of Figure $5 \mathrm{a}$ in $10^{\circ}$ bins of phase. This shows that the phase values over the interval are strongly grouped about a peak in the $170^{\circ}-180^{\circ}$ bin, with $88 \%$ of the data lying within the $80^{\circ}$ phase range between $140^{\circ}$ and $220^{\circ}$. The weighted mean of the phase data within the $0^{\circ}-360^{\circ}$ range is $179^{\circ}$, with a standard deviation of $44^{\circ}$. In these and subsequent related values, the data are weighted according to the inverse of the estimated uncertainties.

[25] With regard to the relationship between the field oscillations and the SKR modulation implied by these results, we note from equation (1) that at SKR modulation maxima (i.e., at times $t$ when $\Phi_{\mathrm{SKR} \text {, s }}(t)=360 \mathrm{~N}$ deg, where $N$ is an integer), the maximum in field component $i$ occurs at azimuth $\varphi_{\max } i$ given (to modulo $360^{\circ}$ ) by

$$
\varphi_{\max i}=-\psi_{\mathrm{Min}, \mathrm{s}} .
$$

The mean $\psi_{\mathrm{Ms}}$ value of $179^{\circ}$ and standard deviation of $44^{\circ}$ found here with respect to the southern SKR phase thus implies that at southern SKR maxima the effective Southern Hemisphere transverse dipole (in-phase with the $r$ component, see Figure 1c) points toward azimuth $-179^{\circ}$ $\pm 44^{\circ}$ (or equivalently $+181^{\circ} \pm 44^{\circ}$ ), i.e., on average tailward and slightly toward dawn at a local time of $0.1 \pm$ $2.9 \mathrm{~h}$. We note that a similar result has been obtained previously for the core region oscillations by Andrews et al. [2008] and Provan et al. [2009a], who found that the quasi-uniform field in this region points radially outward at a local time of $2 \pm 2 \mathrm{~h}$ at southern SKR maxima. As mentioned in section 1, these results thus imply approximate in-phase behavior of the $r$ and $\theta$ com- ponent oscillations between the core and the southern open field region (and with each other) and antiphase behavior of the $\varphi$ component in these regions (being in leading quadrature with $r$ and $\theta$ on southern open field lines and in lagging quadrature in the core). These relationships, in agreement with Provan et al. [2009a], will be examined in greater detail using our full data set in section 3.2 below.

[26] We now consider the results in Figure 5 in more detail, noting that the phase data fall into three main sets, as indicated previously in Figure 4. The first set, marked "A $\mathrm{A}_{\mathrm{S}}$ " in Figure 5 and delimited by vertical dotted lines, corresponds to the first high-latitude mission interval between October 2006 and March 2007 (Revs 30-41), previously studied by Provan et al. [2009a]. In this case, the open field intervals have durations of $\sim 1-3$ days, located at radial distances of $\sim 10-20 \mathrm{R}_{\mathrm{S}}$ in the dawn and dayside sector of the magnetosphere, from which reliable phases can generally be obtained from the filtered data for all three field components. These magnetic phase data exhibit a slow decrease in value across the $\sim 150$ day interval of the data, very similar to the results derived previously by Provan et al. [2009a] with respect to the Kurth et al. [2008] southern SKR modulation phase model, as expected from the discussion of Figure $2 \mathrm{~b}$ in section 2.2. The decreasing phase values are indicative of a small difference in the magnetic and SKR oscillation periods with the magnetic period being shorter than the SKR period. We quantify this difference using a weighted linear least squares fit to these data given by

$$
\psi_{\mathrm{Ms}}(t)=\psi_{\mathrm{Ms} 0}+\psi_{\mathrm{Ms} 1} t
$$

shown by the black solid line in interval $A_{S}$ in Figure 5a. The corresponding period of the magnetic oscillations is then given by

$$
\begin{aligned}
\tau_{M}(t) & =\frac{360}{\left(\frac{\mathrm{d}}{\mathrm{d} t}\left(\Phi_{\mathrm{SKR \textrm {s }}}(t)-\psi_{\mathrm{Ms}}(t)\right)\right)}=\frac{360}{\left(\frac{\mathrm{d} \Phi_{\mathrm{SKRs}}}{\mathrm{d} t}-\psi_{\mathrm{Ms} 1}\right)} \\
& \approx \tau_{\mathrm{SKRs}}\left(1+\frac{\psi_{\mathrm{Ms} 1} \tau_{\mathrm{SKRs}}}{360}\right)
\end{aligned}
$$

where all phases are expressed in degrees. The phase gradient of the fit is $\psi_{\mathrm{Ms} 1}=-0.760 \pm 0.014 \mathrm{deg}_{\text {day }}{ }^{-1}$, and since $\tau_{\text {SKR s }} \approx 10.83 \mathrm{~h}$ during this interval (Figure $5 \mathrm{~b}$ ), we find from equation (5) that the magnetic oscillation period is shorter than the southern SKR modulation period by $\sim 37 \mathrm{~s}$. The value of $\tau_{\mathrm{M}}(t)$ given by equation (5) is plotted as the black line during interval $A_{S}$ in Figure $5 b$, essentially representing the southern SKR period reduced by this amount. It is evident that the magnetic period is still close to the southern SKR period and far removed from the period of the northern SKR modulations. An oscillation at the northern period would result in a negative phase gradient relative to the southern SKR phase of $\sim 15-20 \mathrm{deg} \mathrm{d}^{-1}$, a factor of $\sim 25$ larger than that found here during interval $\mathrm{A}_{\mathrm{S}}$.

[27] The second set of Southern Hemisphere open field phase data, marked " $\mathrm{B}_{\mathrm{S}}$ " in Figure 5, was obtained between June and November 2008 (Revs 74-94) during the first part of the second high-latitude mission interval. These measurements were obtained from shorter $\sim 0.5$ day intervals at 
smaller $\sim 5-10 \mathrm{R}_{\mathrm{S}}$ radial ranges while again spanning dawn between midnight and mid-morning local time. Phase values are determined from the $\varphi$ component only (due to ring current effects in the $r$ and $\theta$ components) and exhibit significantly larger scatter and correspondingly larger uncertainties than in interval $A_{S}$. Nevertheless, these data are still distinctly banded in-phase over a similar range of phases as in interval $A_{S}$. They also form a near-continuous series of measurement with those from the third set of Southern Hemisphere phase data, marked " $\mathrm{C}_{\mathrm{S}}$ " in Figure 5. These were obtained between November 2008 and June 2009 (Revs 95-112) during the second part of the second high-latitude mission interval. Similar to interval $\mathrm{A}_{\mathrm{S}}$, these measurements were obtained from longer $\sim 1-3$ day intervals at $\sim 10-20 \mathrm{R}_{\mathrm{S}}$ radial distances, centered in the postmidnight sector (see, e.g., Figure 3 for Rev 95). These data again produce relatively unscattered phase measurements from all three field components, with near-constant values around $\sim 180^{\circ}$ over the whole interval. A linear fit to the data in intervals $B_{S}$ and $C_{S}$, shown by the black solid line in Figure 5a, has a phase gradient of $\psi_{\mathrm{Ms}}=$ $+0.211 \pm 0.004 \mathrm{deg} \mathrm{d}^{-1}$. From equation (5) this implies an oscillation period that is $\sim 26 \mathrm{~s}$ longer than the southern SKR modulation period, as shown by the corresponding black line in Figure $5 \mathrm{~b}$.

[28] We note that in this and other related least squares linear fits to the phase values, significant outliers in the modulo $360^{\circ}$ data are present, which necessitates the use of a robust fitting algorithm. Here we have employed the iterative RANSAC algorithm with an outlier "threshold" of $90^{\circ}$ [Fischler and Bolles, 1981], such that noticeably discrepant points do not influence the least squares parameters. Further details are provided in Appendix A. Outliers removed in this way are indicated by appropriately colored crosses in Figure 5a (i.e., points with no central dot), although all the data are shown in the adjacent histogram. Typically, this results in the removal of $\sim 3-7$ points from the fits in each interval ( $\sim 7 \%$ of the total), depending on the scatter in the data, although none were removed from the less scattered data in interval $A_{S}$.

[29] The data in Figure 5 thus principally demonstrate that the magnetic field oscillations on southern open field lines are closely tied to the Southern Hemisphere SKR modulations, with $\sim 88 \%$ of the phase data lying within a $\sim 80^{\circ}$ band of phase centered near $\sim 180^{\circ}$. We note that an overall phase difference of at most $\sim 80^{\circ}$ over a $\sim 2.5$ year interval indicates that the two periods differ on average by at most $\sim 4 \mathrm{~s}$ (i.e., by at most $\sim 0.01 \%$ ). Nevertheless, it is also seen that extended intervals of near-monotonic phase drift can also occur, both positive and negative, indicative of differences in period over such shorter intervals of a few tens of seconds as indicated above. Given the intermittent nature of the Southern Hemisphere magnetic phase determinations available, the question then arises of how long each of these intervals can last and thus how large the overall "slip" in-phase can become. The data in Figure 5 suggest durations of at least $\sim 200$ days, with overall "slips" of at least $\sim 90^{\circ}$. We will return to this issue in section 3.2, where measurements of the magnetic oscillation phase in the core region are presented. As mentioned in section 1, the previous results of Provan et al. [2009a] indicate that the $r$ and $\theta$ component oscillations are in-phase between the core and southern open field lines, while the $\varphi$ component oscillations are in antiphase, such that we should expect agreement and continuity between the core oscillation phase data and the Southern Hemisphere open field phase data presented here.

\subsection{Closed Field Core Region}

[30] Results for the core region of the magnetosphere (dipole $L \leq 8$ ) are shown in Figure 6 in the same format as Figure 5, except that the plot now extends over the full interval considered here from $t=100$ to 2057 days as shown previously in Figure 4, spanning SOI to Rev 116. In conformity with the discussion in section 1 concerning a rotating quasi-uniform field in this region following the results of Andrews et al. [2008] and Provan et al. [2009a] (Figures $1 \mathrm{a}$ and $1 \mathrm{~b}$ ), the $r$ and $\theta$ component phase data in Figure $6 \mathrm{a}$ are again shown as measured, while $90^{\circ}$ has now been subtracted from the $\varphi$ component data to bring them to a common phase (as opposed to $90^{\circ}$ being added in Figure 5), in anticipation of the lagging quadrature of these oscillations. It can be seen that the phases so plotted again have common values for all the field components throughout, thus confirming the expected polarization. In addition, in order to readily examine the relationships between these phase data and those obtained on Southern Hemisphere open field lines, we have replotted the phase data from Figure 5 as gray symbols in Figure 6a, although of course these data are not included in the core phase statistics discussed below.

[31] Once more, the principal result for the core region is that the phase data in Figure 6a are clearly banded in-phase throughout the $\sim 5$ years of the study, showing that the averaged period of the dominant oscillations in this region is very close to that of the southern SKR modulations. This is again demonstrated by the histogram plotted to the right of Figure $6 \mathrm{a}$, in which $\sim 73 \%$ of the data fall within the $110^{\circ}$ band between $90^{\circ}$ and $200^{\circ}$, centered on $145^{\circ}$. The weighted average of the $0^{\circ}-360^{\circ}$ data is $147^{\circ}$, with a standard deviation of $55^{\circ}$. An overall phase difference of at most $\sim 110^{\circ}$ over the $\sim 5$ year interval now implies an averaged difference in period between the core field oscillations and the southern SKR modulations of at most $\sim 3 \mathrm{~s}$, again corresponding to $\sim 0.01 \%$. From equation (3), the mean value of the phase and the standard deviation imply that at southern SKR maxima the quasi-uniform equatorial field points radially outward at azimuth $-147^{\circ} \pm 55^{\circ}$ or equivalently $+213^{\circ} \pm 55^{\circ}$, corresponding to a LT of $2.2 \pm 3.7 \mathrm{~h}$, consistent with the previous results of Andrews et al. [2008] and Provan et al. [2009a] mentioned in sections 1 and 3.1. The phase data in Figure 6a also demonstrate a close correspondence between the phases of the oscillations in the core region and those on southern open field lines. Together, they provide evidence of slow secular changes in the relative phase over the interval, both positive and negative within the above overall limits, indicative of extended intervals in which small differences in the magnetic and southern SKR oscillation periods occur, as will be quantified below.

[32] Thus examining the core region data in somewhat more detail, we note that in this case the data can be divided into four sets according to the overview shown in Figure 4. The first two, labeled " $\mathrm{A}_{\mathrm{C}}$ " and " $\mathrm{B}_{\mathrm{C}}$ " in Figure 6 (Revs SOI to 29 and 43 to 54 , respectively), correspond to the first two 


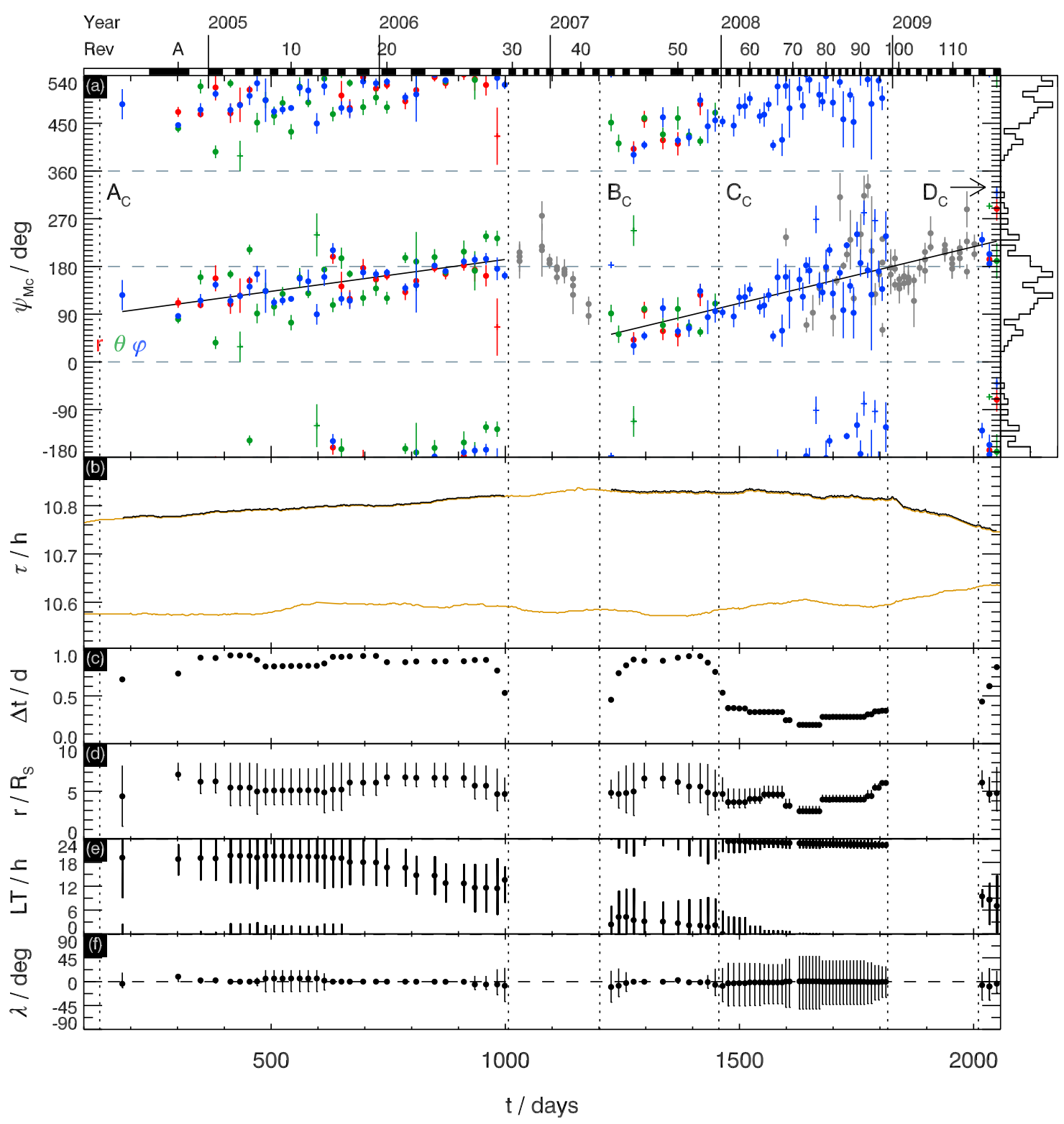

Figure 6. Plot showing oscillation phase results for the core region of closed field lines in a format similar to Figure 5, but now spanning the full interval of the study from $t=100-2057$ days as in Figure 4. The $r$ (red) and $\theta$ (green) component phases are shown as measured as in Figure 5a-f, while since the $\varphi$ (blue) component oscillations are in lagging quadrature, $90^{\circ}$ has been subtracted from these values to bring them to a common phase with the $r$ and $\theta$ component data. In addition, phase values for the Southern Hemisphere open field region shown in Figure 5a are replotted in Figure 6a here as gray symbols for comparison with the core region data shown by the colored symbols.

near-equatorial orbit phases of the Cassini mission studied by Andrews et al. [2008] and Provan et al. [2009a], in which the spacecraft made $\sim 1$ day passes through the core region (as defined here), centered in the dusk and dayside sector for interval $A_{C}$, and in the postmidnight sector for interval $B_{C}$. Relatively unscattered phase values can generally be obtained from the filtered data for all three field components. The third set, labeled " $\mathrm{C}_{\mathrm{C}}$ " in Figure 6 (Revs 55-97), corresponds to the first part of the second high-latitude phase of the mission, in which the spacecraft made rapid north-south transits through the near-midnight core region at radial distances of $\sim 4-5 \mathrm{R}_{\mathrm{S}}$, as illustrated in
Figure 3 for Rev 95, from which more scattered phase values have been obtained from unfiltered $\varphi$ component data only. Nevertheless, these data are still clearly banded in-phase and are consistent with and continue the trend of the phase data in near-equatorial interval $B_{C}$. The fourth set, labeled " $D_{C}$ " in Figure 6 (Revs 114-116), then corresponds to three Revs at the end of the second high-latitude mission phase as the spacecraft returned to lower latitudes.

[33] Overall, the core phase data for intervals $A_{C}$ and $\mathrm{B}_{\mathrm{C}}-\mathrm{D}_{\mathrm{C}}$ both show slow upward drifts with time over a similar band of phase values. This drift occurs over an interval of $\sim 700$ days during interval $\mathrm{A}_{\mathrm{C}}$, in conformity 


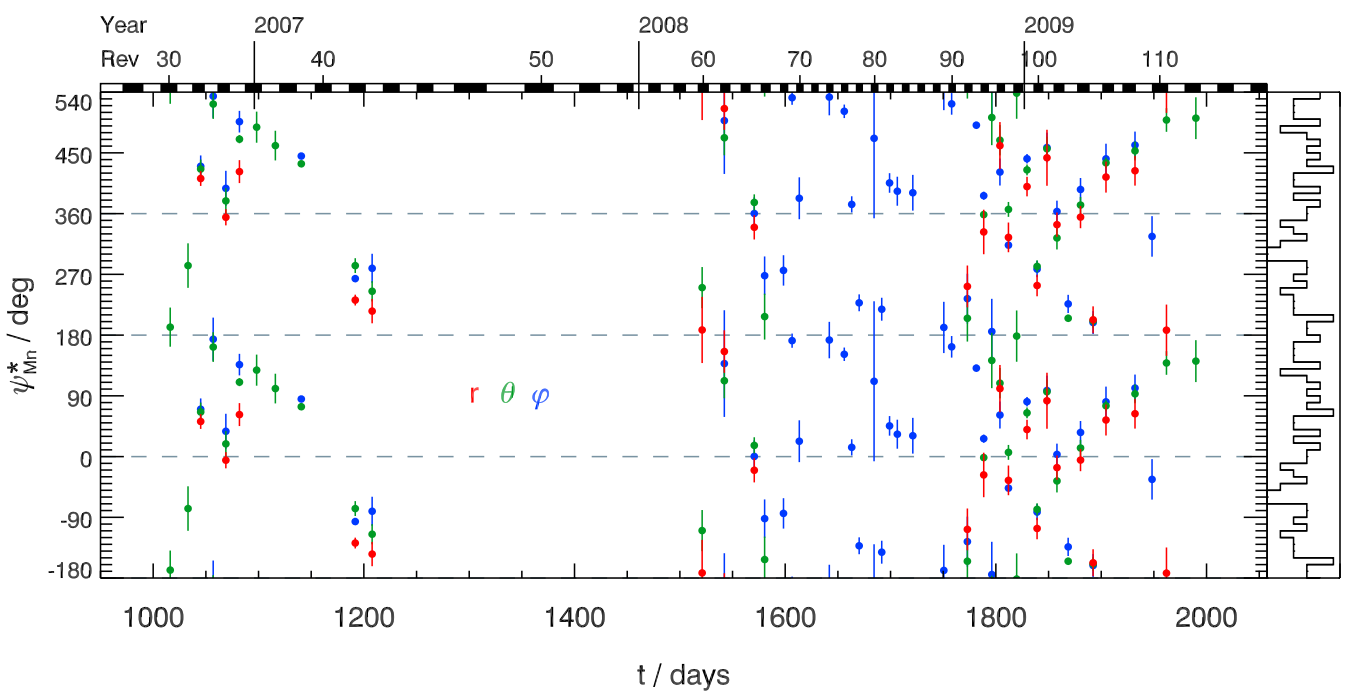

Figure 7. Plot showing the magnetic oscillation phases on Northern Hemisphere open field lines relative to the Southern Hemisphere SKR modulation phase, in a similar format and over the same interval as Figure 5a. The $r$ (red) component phases are shown as measured, while $90^{\circ}$ has been added to the leading quadrature $\varphi$ (blue) component data, and $180^{\circ}$ to the antiphase $\theta$ (green) component data, to bring them to a common phase with the $r$ component data, as seen in the plot. Corresponding ancillary data are shown in Figure 8.

with the prior results of Andrews et al. [2008] and Provan et al. [2009a], and over $\sim 800$ days during intervals $\mathrm{B}_{\mathrm{C}}-\mathrm{D}_{\mathrm{C}}$. Individual linear fits to these data, shown by the black lines in Figure $6 \mathrm{a}$, yield gradients of $\psi_{\mathrm{Ms} 1}=+0.120 \pm$

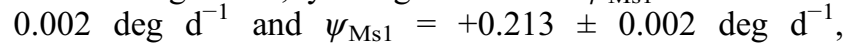
respectively, implying that the magnetic oscillations have a slightly longer period than that of the southern SKR modulations, by $\sim 6$ and $\sim 10 \mathrm{~s}$, respectively. These differences are barely perceptible in the separation between the black and southern period yellow lines in Figure 6b.

[34] We now compare these core region data with the Southern Hemisphere open field phase data replotted as the gray symbols in Figure 6a. Despite the fact that the averaged values of these two data sets differ by $32^{\circ}\left(147^{\circ}\right.$ for the core region data and $179^{\circ}$ for the Southern Hemisphere data), it can nevertheless be seen that these data sets agree closely with each other over the interval and together form a continuous set of combined data. Specifically, the rapidly falling phase data from southern open field interval $A_{S}$ clearly span across the gap between core intervals $A_{C}$ and $B_{C}$, joining the higher phase values at the end of interval $A_{C}$ to the lower phase values at the start of interval $\mathrm{B}_{\mathrm{C}}$. Similarly, the simultaneous core and southern open field data corresponding to intervals $C_{C}$ and $B_{S}$, although with considerable scatter, both show similar rising trends over the same range of phase values, while the subsequent southern data from interval $C_{S}$ are seen to continue this rising trend toward the few core field data points in interval $D_{C}$. We note that the two separate linear fits to the southern data from the combined intervals $\mathrm{B}_{\mathrm{S}}$ and $\mathrm{C}_{\mathrm{S}}$ and the core data from combined intervals $\mathrm{B}_{\mathrm{C}}-\mathrm{D}_{\mathrm{C}}$ are highly comparable, having identical gradients $\left(\psi_{\mathrm{Ms} 1}=+0.211 \pm 0.004 \mathrm{deg} \mathrm{d}^{-1}\right.$ and $\psi_{\mathrm{Ms} 1}=$ $\left.+0.213 \pm 0.002 \mathrm{deg} \mathrm{d^{-1 }}\right)$ and intercepts $\left(\psi_{\mathrm{Ms} 0}=149 \pm 8^{\circ}\right.$ and $\psi_{\mathrm{Ms} 0}=151 \pm 4^{\circ}$, respectively), within determined errors.

[35] This close correspondence between the two data sets thus confirms the phase relationship between the dominant oscillations in the core region and the field oscillations on Southern Hemisphere open field lines over this interval, with $r$ and $\theta$ component oscillations in-phase and $\varphi$ component oscillations in antiphase, with both data sets showing slow drifts in-phase relative to the southern SKR modulations that are clearly linked. The modest $32^{\circ}$ difference in the mean phases of these data sets noted above thus appears to be related to the specific intervals over which these data were obtained relative to the long-term drifts. Combining the data sets yields an overall mean phase value of $157^{\circ}$ and a standard deviation of $54^{\circ}$, which we suggest form the best overall estimates of the mean phase and its range for the core region and southern open field region taken together, relative to the southern SKR phase. These values imply that at southern SKR maxima the core region quasi-uniform field and effective southern open region transverse dipole point radially outward at $1.5 \pm 3.6 \mathrm{~h} \mathrm{LT}$. We also note that a phase shift between these magnetic oscillations and the southern SKR modulations of at most $\sim 110^{\circ}$ during the $\sim 5$ years of the study implies an overall difference in period of at most $\sim 3$ s (i.e., $0.01 \%$ of the period).

[36] It should be noted, however, that the above $\pm 54^{\circ}$ "uncertainty" estimate in the relative phase is not simply due to scatter in the magnetic phase measurements but that the systematic phase drifts evident in Figure 6 also provide a significant contribution. These drifts take the form of two slow rises in-phase with gradients of $\sim 0.120$ and $\sim 0.213 \mathrm{deg} \mathrm{d}^{-1}$ each over $\sim 800$ days, thus spanning $\sim 100^{\circ}$ and $\sim 180^{\circ}$, joined by a more rapid decrease of $\sim 150^{\circ}$ over $\sim 200$ days occurring principally during the first high-latitude mission interval. Fitting some suitable curve through these data in future work would yield an overall core and Southern Hemisphere magnetic phase model with significantly reduced relative scatter in the data points. The origin of these phase drifts remains unknown at the present time and is also a topic for future study. Possible SKR 


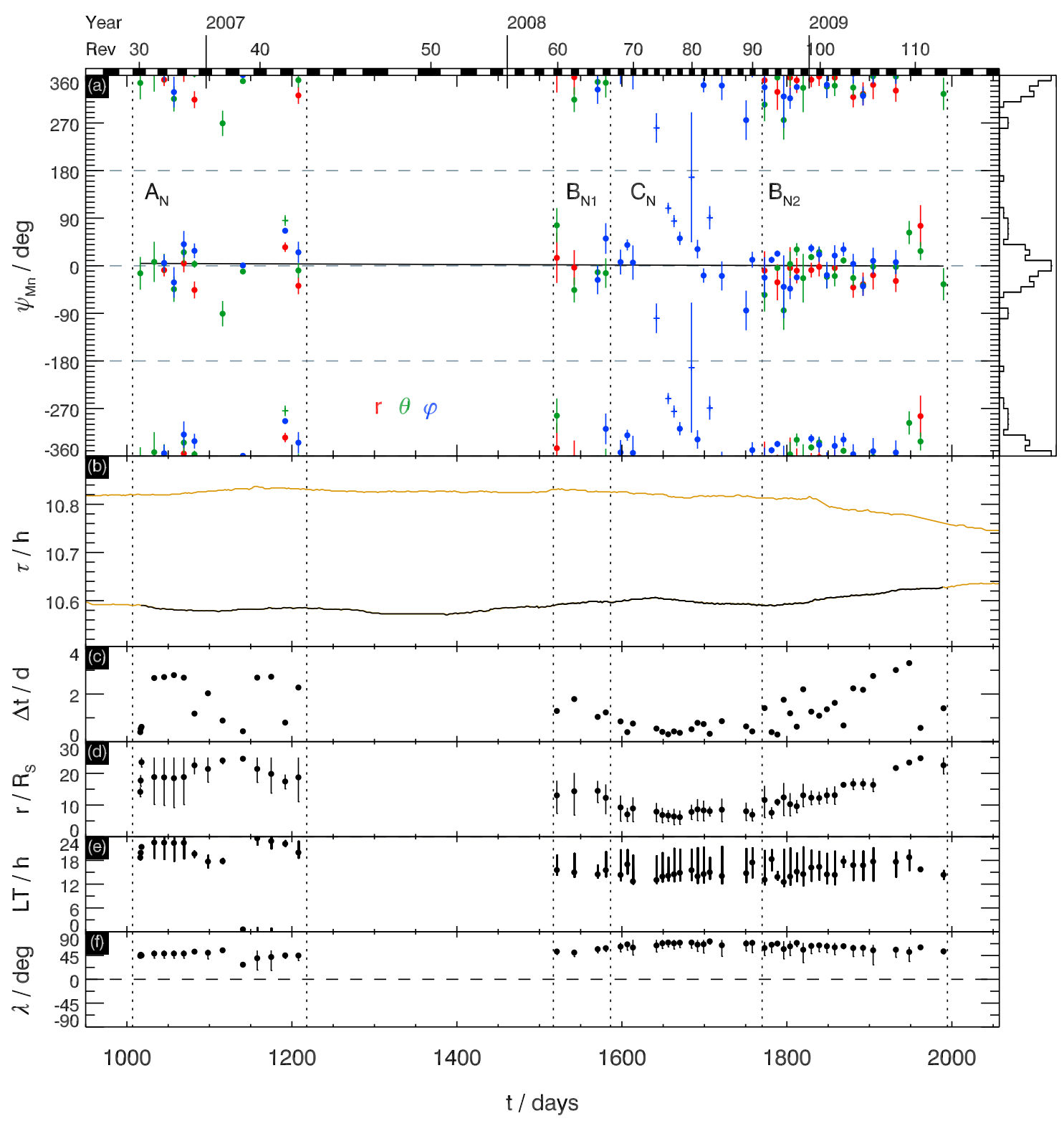

Figure 8. Plot showing the magnetic oscillation phases on Northern Hemisphere open field lines relative to the Northern Hemisphere SKR modulation phase, in a similar format and over the same interval as Figure 5. As in Figure 7, the $r$ (red) component phases are shown as measured, while $90^{\circ}$ has been added to the leading quadrature $\varphi$ (blue) component data, and $180^{\circ}$ to the antiphase $\theta$ (green) component data, to bring them to a common phase with the $r$ component data. We also note that in Figure 8a we have shifted the two-cycle phase range to between $-360^{\circ}$ and $+360^{\circ}$ in order to centrally accommodate the banded phase data.

visibility issues connected with spacecraft orbital position remain conjectural.

\subsection{Northern Hemisphere Open Field Lines}

[37] We now turn to the oscillations on open field lines in the Northern Hemisphere, and given the above results, we first examine whether the phases of these oscillations are also ordered by the Southern Hemisphere SKR modulation phase, as was (in effect) investigated previously by Provan et al. [2009a]. In Figure 7, we show these results in the same format and over the same restricted interval covering the two high-latitude mission phases as employed for the corre- sponding Southern Hemisphere results in Figure 5a (ancillary position data will be shown later in Figure 8). In anticipation of the planet-centered rotating dipole polarization found by Provan et al. [2009a], while the phase of the $r$ component data is again shown as measured, as in Figures 5 and 6, in this case $90^{\circ}$ has been added to the phase of the $\varphi$ component (in leading quadrature with $r$ for a rotating transverse dipole) and $180^{\circ}$ to the phase of the $\theta$ component (in antiphase with $r$ in the Northern Hemisphere for a rotating transverse dipole), to bring all the phase data to a common value (see Figures 1c and 1d). It can be seen that these shifts generally do bring the phase data successfully together for 
each Rev, thus confirming the polarization results of Provan et al. [2009a] for this larger northern data set.

[38] It can also be seen in Figure 7 that the data corresponding to the first high-latitude mission interval (Revs 30-43) are somewhat grouped in-phase, as reported by Provan et al. [2009a], although comparison with Figures 5 and 6 shows that they are not continuous with the Southern Hemisphere and core data, as would be expected, for example, if the northern field oscillations were due to the same effective rotating transverse dipole as observed in the south (which would give $r$ component oscillations in the two hemispheres in-phase throughout). Furthermore, the later data newly examined here (Revs 60-112) show no consistent banding in-phase, as is clearly evident in the histogram shown on the right of the plot, which indicates instead that the data overall are distributed almost uniformly across a complete cycle. We thus conclude that the grouping previously reported by Provan et al. [2009a] is essentially accidental and that there is no overall organization of the northern open region oscillation phases by the southern SKR modulation phase.

[39] We thus consider instead whether the northern open field phase data are organized by the northern SKR modulation phase, noting that such organization is not inconsistent with the scattered results with respect to the southern SKR phase seen in Figure 7. As indicated in section 3.1, the phase of an oscillation at the northern SKR period shifts by $\sim 15-20 \mathrm{deg} \mathrm{d}^{-1}$ relative to the phase of the southern SKR period (and vice versa), leading to Rev-to-Rev shifts inphase of a large fraction of a full cycle over the 10-20 day intervals between Revs. Consistent shifts of such magnitude are not discernable in Figure 7. We note that if the Southern Hemisphere open field data or core region data shown in Figures 5 and 6 are similarly analyzed relative to the northern SKR phase, the results (not illustrated here) also show scattered behavior similar to that in Figure 7.

[40] In Figure 8, we thus show the phases of the magnetic field oscillations on Northern Hemisphere open field lines relative to the northern SKR modulation phase, in a similar format, and over the same interval, as in Figures 5 and 7. The phase shifts applied to the individual component data to bring them to a common value are the same as indicated above for Figure 7, corresponding to a planet-centered rotating transverse dipole, with the $r$ component phase (having the same phase as the effective transverse dipole) being as measured. It can be seen that the phase data are now strongly banded, with $76 \%$ of the data lying in the $90^{\circ}$ phase band between $-50^{\circ}$ and $+40^{\circ}$ centered near $0^{\circ}$. We note that, compared with Figures 5 and 6 , we have shifted the two-cycle phase range in Figure $7 \mathrm{a}$ to between $-360^{\circ}$ and $+360^{\circ}$ in order to centrally accommodate these banded data. This result then demonstrates that the period of the northern magnetic field oscillations is closely similar to that of the northern SKR modulation, with a phase difference of at most $\sim 90^{\circ}$ over the $\sim 3$ year interval implying a overall difference in period of at most $\sim 4$ s (i.e., $\sim 0.01 \%$ ).

[41] With regard to the value of the phase in Figure 8, we note that the weighted average phase of the $-180^{\circ}$ to $+180^{\circ}$ data is $6^{\circ}$, with a standard deviation of $36^{\circ}$. Recalling from equation (3) that the effective dipole, in-phase with the $r$ component, points toward azimuth $-\psi_{\mathrm{Mn}}$ at northern SKR maxima, the latter result implies that the northern dipole points sunward on average and slightly toward dawn, at $11.7 \pm 2.4 \mathrm{~h} \mathrm{LT}$, at northern SKR maxima. Thus, while the southern period quasi-uniform field and high-latitude effective dipole point down tail (and toward dawn) at southern SKR maxima, the northern period high-latitude effective dipole points sunward (and also slightly toward dawn) at northern SKR maxima.

[42] As with the Southern Hemisphere open field and core regions examined above, the phase data for the Northern Hemisphere open field region can again be divided into three groups with differing characteristics depending on the nature of the Cassini orbit, as overviewed in Figure 4. The first group marked " $\mathrm{A}_{\mathrm{N}}$ " in Figure 8 (Revs 30-43) corresponds to the first high-latitude mission phase previously studied by Provan et al. [2009a]. This contains extended $\sim 1-3$ day passes through the northern open region at radial distances in the range $\sim 10-25 \mathrm{R}_{\mathrm{S}}$ centered in the premidnight sector, from which phase data can generally be obtained for all three field components. The second and third groups correspond to the second high-latitude mission phase shown in Figure 4. These data can broadly be divided into earlier and later intervals marked " $\mathrm{B}_{\mathrm{N} 1}$ " and " $\mathrm{B}_{\mathrm{N} 2}$ " (Revs 60-66 and 92-112) consisting of $\sim 1-2$ day passes at radial distances $\sim 10-20 \mathrm{R}_{\mathrm{S}}$, from which phase data can again generally be obtained for all three field components (see Figure 3 for Rev 95), and a central interval marked " $\mathrm{C}_{\mathrm{N}}$ " (Revs 68-90) consisting of short $\sim 6-24 \mathrm{~h}$ passes at radial distances of $\sim 5-10 \mathrm{R}_{\mathrm{S}}$, from which more scattered data can be obtained only from the $\varphi$ component. The data for intervals $B_{N}$ and $C_{N}$ all span the postnoon and dusk sector in local time. It is thus seen that the nature of the northern open field data are similarly variable as for the southern open field and core region data. Nevertheless, the results in Figure 8 indicate that these form an apparently featureless data set (assuming the variability in interval $C_{N}$ is mainly due to scatter), with little indication of significant long-term drifts in-phase over the $\sim 2.5$ year interval covered like those seen in Figures 5 and 6 . In particular, the weighted average of the phase data for well-separated intervals $A_{N}$ and $\mathrm{B}_{\mathrm{N} 2}$ are $8^{\circ}$ (with a standard deviation of $36^{\circ}$ ) and $-1^{\circ}$ (with a standard deviation of $25^{\circ}$ ), respectively, thus being essentially consistent with each other within the uncertainties. If we then make a weighted linear fit to all these data, shown by the black solid line in Figure 8a, we find an overall gradient of only $-0.005 \pm 0.001 \mathrm{deg} \mathrm{d}^{-1}$. This corresponds to a difference in period between the northern open field oscillations and the northern SKR modulations of only $\sim-0.2$ s (i.e., $0.0005 \%$ ), a difference which is not discernable in Figure 8a.

\section{Summary and Discussion}

[43] In this paper, we have made a first detailed investigation of the phase of the magnetic field oscillations near the planetary rotation period in Saturn's magnetosphere relative to the phases of the separate northern and southern SKR emission modulations, as recently discovered by Gurnett et al. [2009a]. We have employed $\sim 5$ years of magnetic field observations spanning the interval from SOI in July 2004 during Saturn southern summer conditions, to Rev 116 in August 2009 close to vernal equinox, and have compared the magnetic oscillation phases with the SKR modulation 
phases derived from $\mathrm{X}$ mode radio powers from the two hemispheres by Lamy et al. (manuscript in preparation), as described in section 2.2 (see http://www.lesia.obspm.fr/ kronos/guest.php). The results thus build on the previous related studies of Andrews et al. [2008] and Provan et al. [2009a] that examined earlier subsets of the magnetic data in relation to the dominant southern SKR modulations during this interval analyzed by Kurth et al. [2007, 2008]. Here following Provan et al. [2009a], we have examined the oscillations in three distinct magnetospheric regions, corresponding to the high-latitude northern and southern open field regions to radial distances of $25 \mathrm{R}_{\mathrm{S}}$, characterized by the lack of warm or hot plasma, and the quasi-dipolar core region of the magnetosphere within dipole $L \leq 8$, defined so as to exclude the magnetic signatures of field-aligned currents flowing near the boundary between open and closed field lines at all local times.

[44] Our analysis demonstrates that there exists two systems of field oscillations in Saturn's magnetosphere associated with the two SKR periods. The oscillations on southern open field lines and in the quasi-dipolar core region take place near the southern SKR period of $\sim 10.8 \mathrm{~h}$, while the oscillations on northern open field lines take place near the northern SKR period of $\sim 10.6 \mathrm{~h}$. Maximum overall phase shifts between the magnetic field oscillations and the corresponding SKR modulations of $\sim 110^{\circ}$ over $\sim 5$ years in the first case and $\sim 90^{\circ}$ over $\sim 2.5$ years in the second imply maximum overall differences in period of only $\sim 3-4 \mathrm{~s}$, corresponding to $\sim 0.01 \%$ of the respective SKR periods.

[45] Concerning the nature of the oscillatory field, our results show that in the core region the southern period perturbations take the form of a rotating quasi-uniform field in the equatorial plane, with the addition of a colatitudinal field which is such that the perturbation field lines form arches with apices to the north. In other words, the $\varphi$ component oscillates in lagging quadrature with the $r$ component, while the $\theta$ component oscillates in-phase with the $r$ component, as shown previously by Andrews et al. [2008]. The corresponding oscillations on southern open field lines instead have the form of a planet-centered rotating transverse dipole, in other words, the $\varphi$ component now oscillates in leading quadrature with the $r$ component, while the $\theta$ component oscillates in-phase with the $r$ component, as shown previously by Provan et al. [2009a]. The results derived here then show that the rotating quasi-uniform field in the equatorial region and the effective rotating transverse dipole on southern open field lines maintain approximately the same direction as each other over the $\sim 5$ years of our study. In other words, the $r$ and $\theta$ components in the two regions oscillate in-phase at the southern period, while the $\varphi$ components in the two regions oscillate in antiphase.

[46] Overall, these results indicate that the southern period perturbation field lines form loops passing through the equatorial region and closing at southern high latitudes, as sketched in Figure 9a. This shows fields in the principal meridian of the perturbation field at a given instant, as in Figures $1 \mathrm{~b}$ and $1 \mathrm{~d}$, where the black dashed lines show the quasi-static "background" magnetospheric field, with closed field lines at lower latitudes (gray region) and open field lines at high latitudes (clear region) mapping into the northern and southern polar regions. The perturbation field lines of the southern system at this instant are then indicated by the red solid lines, forming arches with apices to the north in the equatorial region and closing over the southern pole in the southern region of open field lines. The effective centered transverse dipole corresponding to the latter field points to the left at the instant depicted in Figure 9a, approximately parallel to the near-equatorial field, as indicated by the central red arrow. To a first approximation, the perturbation field lines out of the plane shown can then be obtained simply by displacing these field loops directly into or out of the plane of Figure 9. This perturbation field pattern then rotates in time approximately rigidly about the central axis at close to the southern SKR modulation period as indicated, giving rise to field oscillations with the relative polarization found in this study. It should be emphasized, however, that although it is difficult to represent the true perturbation field geometry in a simple 2-D sketch, the intention here is to indicate that the equatorial quasi-uniform field region corresponds to the inner region of closed field lines, while the quasi-dipolar field which closes these field loops over the pole corresponds to the region of open field lines. The implied associated currents that flow between these regions will be discussed further below.

[47] Our results for the northern period oscillations on northern open field lines also show that they have the form of a planet-centered rotating transverse dipole, such that the $\varphi$ component oscillates in leading quadrature with the $r$ component, while the $\theta$ component oscillates in antiphase with the $r$ component, as shown previously by Provan et al. [2009a]. The form of this field is shown by the green lines in the north polar region of Figure $9 b$, corresponding to an effective centered transverse dipole pointing to the right at the instant depicted as shown by the green arrow. If these perturbation field lines also close in the equatorial region equivalent to that shown for the southern period oscillation in Figure 9a, the implication is that an additional northern period field oscillation should also be present in the equatorial region as sketched in Figure $9 \mathrm{~b}$. These field oscillations would again have the $\varphi$ component in lagging quadrature with $r$, as for the southern period oscillations, but now the $\theta$ component would be in antiphase with $r$, such that the equatorial field lines form arches with apices to the south. The presence of such oscillations, at amplitudes less than those of the southern period oscillations, is by no means ruled out by the data analyses conducted to date and, indeed, may contribute to the considerable phase "jitter" observed in this region in Figure 6 and noted in section 1 [Andrews et al., 2008], additional to that possibly produced by solar wind-related effects [Zarka et al., 2007].

[48] In addition to determining the form of the oscillatory fields, our results also determine the phase between the magnetic oscillations and the corresponding SKR modulations. In agreement with the previous results of Andrews et al. [2008] and Provan et al. [2009a], we find from our combined core and open field region results that at times of southern SKR maxima, the southern period quasi-uniform equatorial field and the high-latitude southern effective transverse dipole point tailward and on average toward dawn, i.e., radially outward at $1.5 \pm 3.6 \mathrm{~h} \mathrm{LT}$. However, we find that the corresponding high-latitude northern effective transverse dipole and suggested secondary equatorial field point sunward and also on average slightly toward dawn at northern 

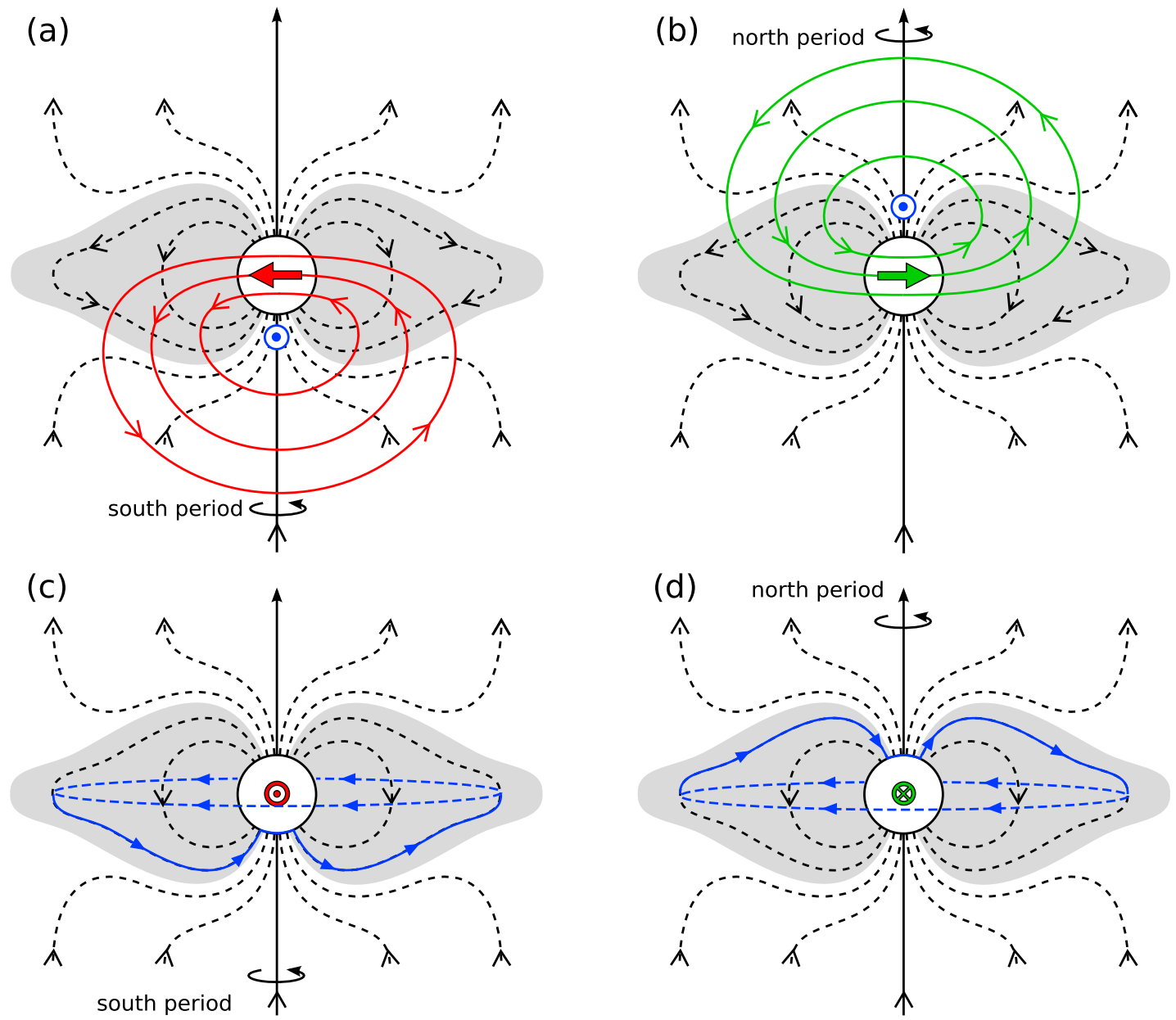

Figure 9. Sketches showing the form of the oscillatory perturbation fields deduced from the results of this study together with the implied current systems. ( $a$ and $b$ ) The principal meridian of the perturbation at a given instant (as in Figures $1 \mathrm{~b}$ and 1d), where the black dashed lines show the quasi-static "background" magnetospheric field, with closed field lines at lower latitudes (gray region) and open field lines at higher latitudes (clear region) mapping into the northern and southern polar regions. The perturbation field lines are then indicated by the colored solid lines, red for the southern system in Figure 9a and green for the northern system in Figure 9b. To a first approximation the perturbation field lines out of the planes shown can be obtained simply by displacing the perturbation field loops directly into or out of the plane of the figure. The direction of the centered transverse dipole corresponding to the quasi-dipolar perturbation field in the open field region is shown by the solid arrow, while the sense of the current associated with the overall field perturbations given by Ampère's law is indicated by the blue symbols, directed out of the plane in both diagrams. The patterns shown correspond to SKR maxima when the Sun is on the right in both panels, such that at these times the associated current flows approximately from dusk to dawn. (c and d) More detailed views of the current systems associated with Figures 9a and 9b, respectively, where both Figures $9 \mathrm{a}$ and $9 \mathrm{~b}$ are viewed from the left. Field-aligned and ionospheric currents flowing from left to right in Figures $9 \mathrm{c}$ and $9 \mathrm{~d}$ are indicated by the solid blue lines, while the return currents flowing from right to left through the equatorial region outside the quasi-dipolar equatorial magnetosphere are indicated by the dashed blue lines. These perturbation field patterns and current systems then rotate independently in time, approximately rigidly about the central axis, at close to the southern SKR period in Figure 9a and close to the northern SKR period in Figure 9b, as indicated.

SKR maxima, i.e., radially outward at $11.7 \pm 2.4 \mathrm{~h} \mathrm{LT}$. Thus, our results indicate that the perturbation fields of the northern system at northern SKR maxima point essentially opposite to the perturbation fields of the southern system at southern SKR maxima. The implications of this for the associated current systems will be discussed further below.
[49] While the above description forms a basic picture of the magnetic field oscillations determined in our study, more subtle effects are also evident. In particular, in common with the results of Andrews et al. [2008] and Provan et al. [2009a], we find that slow variations in the phase of the southern period oscillations are evident relative to the phase of the southern SKR modulations, which appear to be 
present as a consistent phenomenon in both the core region and on southern open field lines. Over the 5 year interval of this study, these take the form of two slow increases in the magnetic phase relative to the southern SKR phase over intervals of $\sim 800$ days, separated by a shorter $\sim 200$ day interval of more rapid decrease, which is such that the increases on either side cover much the same range of relative phase values in each case. The physical origins of these phase drifts remain unknown at the present time. Slow increases in the magnetic phase indicate that the period of the magnetic oscillations is slightly longer than those of the corresponding SKR modulation and vice versa for slow decreases in the magnetic phase. It should be emphasized, however, that the implied differences in period are small, certainly when compared with the $\sim 10$ min difference in period between the southern and northern oscillations (and the secular variations in period observed in both). Specifically, the positive phase gradients during the two slow increases in-phase seen in Figure 6 of $\sim 0.120$ and $\sim 0.213 \mathrm{deg}^{-1}$ imply differences in period of only $\sim 6$ and $\sim 10$ s (i.e., $\sim 0.02$ and $\sim 0.03 \%$ of the southern period), while the negative phase gradient during the interval of more rapid phase decrease that occurs at $\sim-0.760 \mathrm{deg}^{-1}$ implies a difference in period of 37 s (i.e., $\sim 0.10 \%$ of the southern period). We note that the overall peak-to-peak "slip" in the relative phase associated with this phenomenon is $\sim 150^{\circ}$, this effect being a significant contributor to the error estimate (standard deviation) quoted above for the direction of the equatorial quasi-uniform field and southern transverse dipole at southern SKR maxima. Results for the northern period oscillations, however, indicate that the relative phase drifts are significantly smaller than for the southern period system, with no long-term trends with magnitudes larger than $\sim 0.1 \mathrm{deg} \mathrm{d}^{-1}$ being discernable throughout.

[50] With regard to the origins of the magnetic field effects investigated here, our results clearly indicate the presence of two physically distinct current systems that rotate with different periods in the two hemispheres. This is already evident in Figures 9a and 9b, where Ampère's law applied to the perturbation field loops imply rotating currents, which flow out of the plane of the diagram in both cases at the instants depicted, as indicated very schematically by the blue symbols shown in Figures 9a and 9b. The current systems envisaged are indicated in more detail in Figures 9c and 9d, which correspond to Figures 9a and 9b, respectively, each viewed from the left. Following the prior discussions of Southwood and Kivelson [2007] and Provan et al. [2009a], we envisage the presence of field-aligned currents flowing from left to right in both cases as shown by the blue solid lines, located in the interface between the regions of quasi-uniform and quasi-dipolar oscillatory perturbation field and passing centrally through the polar ionosphere. These currents then reach the equatorial plane on outer closed field lines, bounding the region occupied by the rotating quasi-uniform field to the central quasi-dipolar (core) region of the magnetosphere, within equatorial radial distances of $\sim 15 \mathrm{R}_{\mathrm{S}}$ according to the results of Andrews et al. [2010]. These currents must then close in the equatorial magnetosphere beyond the core region, as indicated schematically by the dashed blue lines. It may be noted that these overall current systems indeed have a magnetic moment in the sense depicted in Figures 9a and 9b, as also indicated by the red and green symbols in Figures $9 \mathrm{c}$ and $9 \mathrm{~d}$. The current systems in the two hemispheres then rotate independently with different periods, giving rise to oscillations dominated by the corresponding period at high-latitudes on open field lines, while potentially superposing in the equatorial region as sketched in Figures 9a and 9b. Our results show, however, that the southern period current system and related oscillations are dominant in the core region throughout the preequinox southern summer interval investigated here, perhaps due to higher conductivities and currents in the southern summer ionosphere. We may then speculate that dominance could shift to northern period oscillations in the equatorial region some time postequinox. Continued study of the postequinox oscillations will undoubtedly yield further insight into the origins of these phenomena.

[51] As indicated in section 1, SKR emissions are believed to be generated by accelerated auroral electrons via the cyclotron maser instability and may thus be expected to be associated both with the UV auroral oval [Lamy et al., 2009] and regions of strong upward-directed field-aligned current [Lamy et al., 2010]. The implications of the phasing of the magnetic oscillations in the two hemispheres with respect to the SKR modulations is then such that the SKR emission maxima in both cases occur when the rotating upwarddirected field-aligned current region passes through the dawn sector in the two hemispheres, due possibly to an interaction between the rotating current systems discussed here and quasi-static currents set up, e.g., by the solar wind interaction with the magnetosphere [e.g., Galopeau et al., 1995; Cecconi and Zarka, 2005; Southwood and Kivelson, 2009]. The senses of the perturbation fields and currents in Figures 9 thus show the condition for SKR maxima in the two hemispheres if the Sun is located to the right in each of the Figures $9 \mathrm{a}$ and $9 \mathrm{~b}$ and into the plane of the plots in Figures $9 \mathrm{c}$ and $9 \mathrm{~d}$. The region of downward-directed fieldaligned current on the opposite side of the planet then plausibly forms the rotating source of upward-directed auroral hiss, in agreement with the phasing of the latter in the two hemispheres reported by Gurnett et al. [2009b]. We note that Nichols et al. [2010] have indeed recently shown that Saturn's UV auroras brighten in the dawn side at the corresponding SKR maxima in each hemisphere, with some indication of a weaker antiphase effect in the auroras on the dusk side, consistent with the effect of the rotating upward currents envisaged here. We also note that the periodic oscillations of the plasma sheet in the outer magnetosphere and tail, discussed previously in terms of the effect of a rotating transverse dipole formed by a rotating external current system [e.g., Southwood and Kivelson, 2007; Provan et al., 2009a; Andrews et al., 2010], must now be augmented to include two effective transverse dipoles that rotate with differing periods, whose absolute and relative strengths may plausibly depend on Saturn's seasons. Such considerations may then provide an explanation for the dual periodicity in the fluxes of energetic electrons in Saturn's outer magnetosphere reported by Carbary et al. [2009b].

[52] We finally note that while the rotating quasi-uniform field in the core region, directed approximately transverse to the equatorial planetary field, produces both tilting of the field lines and rocking of the magnetic equator as just indicated, the oscillations in the colatitudinal field component, near-parallel to the equatorial planetary field, are instead associated with rotating changes in the field strength. 
Related rotating changes in the plasma pressure are also known to occur, as indicated by the studies of Burch et al. [2009] and Carbary et al. [2009a, 2010b], that must combine with the field pressure changes to cause rotating changes in the total pressure that modulate the positions of both the magnetopause and bow shock [Clarke et al., 2010a, 2010b]. These latter studies relate specifically to the southern period oscillations that are dominant in the nearequatorial region during the preequinox interval analyzed here, but in view of the results found here it seems reasonable to suppose that weaker pressure oscillations at the northern period are also simultaneously present. In addition to the transverse perturbations associated with rotating fieldaligned currents therefore it thus appears that rotating compressional oscillations are also simultaneously present at the two periods. It remains to be determined how these oscillation systems are maintained at periods that not only differ from each other, but also from that of the subcorotating and drifting plasma.

\section{Appendix A: The RANSAC Algorithm}

[53] In this appendix we provide a brief overview of the RANSAC (RANdom SAmple Consensus) algorithm [Fischler and Bolles, 1981] employed here to determine linear fits to the magnetic phase data shown in Figures 5, 6, and 8. Unmodified least square algorithms are not suitable for fitting these data since they contain substantial outliers, which significantly influence the fitted parameters. The RANSAC algorithm removes these outliers through an iterative process that searches for a best "consensus set" within the data to be fitted, removing the most significant outliers and yielding an unbiased fit. From the input set of data to be fitted, a random subset of a given size is selected, which may or may not contain outlying values as well as "true" measurements, and fit parameters are estimated from this subset using least squares. Each remaining data point is then inspected and included in the subset if the difference between the data value and the corresponding fit value is less than some threshold $\delta$. After this operation, provided that the total number of points in the subset is now larger than some required fraction $f$ of the original input data set, a "consensus data set" has been found. The least squares linear fit parameters are then reevaluated from this consensus set. This procedure is repeated many times for differing randomly chosen data sets of the same initial size, and the results were ranked by use of a "cost function" equal to the modular difference between the data values and the fit to the consensus data set, summed over all of the data, but where the difference is limited to the value $\delta$. The procedure is stopped when no further decrease in the cost function is obtained after a given number of trials, the latter in practice typically being several thousand.

[54] Employed in this manner, the algorithm is not particularly sensitive to the choice of fraction $f, 70 \%$ of the total data set being employed here, and is typically stable for reasonable values of $\delta$. The choice of $\delta$, which determines whether a given data point is an outlier with respect to a given fit, must generally be chosen using some a priori knowledge of the data set to be fitted. Here we have set $\delta=90^{\circ}$, such that points more than $\pm 25 \%$ of a cycle adrift are flagged as outliers. Our prime concern is then the initial number of points in the randomly selected subset. Too many points, and the likelihood of any given subset containing one or more outliers increases, meaning that the algorithm must be run for longer to produce reliable results. Too few, and the intrinsic scatter in the data results in a poor model and subsequent valid measurements are rejected, such that the end result is again unreliable. Here we have chosen this parameter to be $40 \%$ of each data set, which is found to produce consistent and reliable results.

[55] Acknowledgments. Work at Leicester was supported by STFC grants PP/E000983/1 and ST/H002480/1. A.J.C. was supported by STFC funding to MSSL/UCL, L.L. and M.K.D. were supported by STFC funding to Imperial College, and D.J.A. was supported by a STFC Quota Studentship. We thank S. Kellock and the Cassini team at Imperial College for access to processed magnetic field data, B. Cecconi at LESIA for assistance with SKR data processing, and G.R. Lewis and L.K. Gilbert at MSSL for CAPS/ELS data processing.

[56] Masaki Fujimoto thanks the reviewers for their assistance in evaluating this paper.

\section{References}

Andrews, D. J., E. J. Bunce, S. W. H. Cowley, M. K. Dougherty, G. Provan, and D. J. Southwood (2008), Planetary period oscillations in Saturn's magnetosphere: Phase relation of equatorial magnetic field oscillations and SKR modulation, J. Geophys. Res., 113, A09205, doi:10.1029/ 2007JA012937.

Andrews, D. J., S. W. H. Cowley, M. K. Dougherty, and G. Provan (2010), Magnetic field oscillations near the planetary period in Saturn's equatorial magnetosphere: Variation of amplitude and phase with radial distance and local time, J. Geophys. Res., 115, A04212, doi:10.1029/2009JA014729.

Arridge, C. S., N. André, N. Achilleos, K. K. Khurana, C. L. Bertucci, L. K. Gilbert, G. R. Lewis, and M. K. Dougherty (2008), Thermal electron periodicities at $20 \mathrm{R}_{\mathrm{S}}$ in Saturn's magnetosphere, Geophys. Res. Lett., 35, L15107, doi:10.1029/2008GL034132.

Bunce, E. J., S. W. H. Cowley, I. I. Alexeev, C. S. Arridge, M. K. Dougherty, J. D. Nichols, and C. T. Russell (2007), Cassini observations of the variation of Saturn's ring current parameters with system size, J. Geophys. Res., 112, A10202, doi:10.1029/2007JA012275.

Burch, J. L., A. D. DeJong, J. Goldstein, and D. T. Young (2009), Periodicity in Saturn's magnetosphere: Plasma cam, Geophys. Res. Lett., 36, L14203, doi:10.1029/2009GL039043.

Carbary, J. F., and S. M. Krimigis (1982), Charged particle periodicity in the Saturnian magnetosphere, Geophys. Res. Lett., 9(9), 1073-1076, doi:10.1029/GL009i009p01073.

Carbary, J. F., D. G. Mitchell, S. M. Krimigis, and N. Krupp (2007a), Electron periodicities in Saturn's outher magnetosphere, J. Geophys. Res., 112, A03206, doi:10.1029/2006JA012077.

Carbary, J. F., D. G. Mitchell, S. M. Krimigis, and N. Krupp (2007b), Evidence for spiral pattern in Saturn's magnetosphere using the new SKR longitudes, Geophys. Res. Lett., 34, L13105, doi:10.1029/2007GL030167.

Carbary, J. F., D. G. Mitchell, S. M. Krimigis, D. C. Hamilton, and N. Krupp $(2007 \mathrm{c})$, Spin period effects in magnetospheres with no axial tilt, Geophys. Res. Lett., 34, L18107, doi:10.1029/2007GL030483.

Carbary, J. F., D. G. Mitchell, P. Brandt, C. Paranicas, and S. M. Krimigis (2008a), ENA periodicities at Saturn, Geophys. Res. Lett., 35, L07102, doi:10.1029/2008GL033230.

Carbary, J. F., D. G. Mitchell, P. Brandt, E. C. Roelof, and S. M. Krimigis (2008b), Periodic tilting of Saturn's plasma sheet, Geophys. Res. Lett., 35, L24101, doi:10.1029/2008GL036339.

Carbary, J. F., S. M. Krimigis, D. G. Mitchell, C. Paranicas, and P. Brandt (2009a), Energetic neutral atom (ENA) and charged particle periodicities in Saturn's magnetosphere, Adv. Space Res., 44, 483-493, doi:10.1016/ j.asr.2009.04.019.

Carbary, J. F., D. G. Mitchell, S. M. Krimigis, and N. Krupp (2009b), Dual periodicities in energetic electrons at Saturn, Geophys. Res. Lett., 36, L20103, doi:10.1029/2009GL040517.

Carbary, J. F., D. G. Mitchell, S. M. Krimigis, D. A. Gurnett, and W. S. Kurth (2010), Phase relations between energetic neutral atom intensities and kilometric radio emissions at Saturn, J. Geophys. Res., 115, A01203, doi:10.1029/2009JA014519.

Cecconi, B., and P. Zarka (2005), Model of a variable radio period for Saturn, J. Geophys. Res., 110, A12203, doi:10.1029/2005JA011085.

Cecconi, B., L. Lamy, P. Zarka, R. Prangé, W. S. Kurth, and P. Louarn (2009), Gonipolarimetric study of the revolution 29 perikrone using the Cassini Radio and Plasma Wave Science instrument high-frequency 
radio receiver, J. Geophys. Res., 114, A03215, doi:10.1029/ 2008JA013830.

Clarke, K. E., et al. (2006), Cassini observations of planetary period oscillations of Saturn's magnetopause, Geophys. Res. Lett., 33, L23104, doi:10.1029/2006GL027821.

Clarke, K. E., D. J. Andrews, A. J. Coates, and S. W. H. Cowley (2010a), Magnetopause oscillations near the planetary period at Saturn: Occurrence, phase and amplitude, J. Geophys. Res., 115, A08209, doi:10.1029/ 2009JA014745.

Clarke, K. E., D. J. Andrews, A. J. Coates, S. W. H. Cowley and A. Masters (2010b), Magnetospheric period oscillations of Saturn's bow shock J. Geophys. Res., 115, A05202, doi:10.1029/2009JA015164.

Cowley, S. W. H., D. M. Wright, E. J. Bunce, A. C. Carter, M. K. Dougherty, G. Giampieri, J. D. Nichols, and T. R. Robinson (2006), Cassini observations of planetary- period magnetic field oscillations in Saturn's magnetosphere: Doppler shifts and phase motion, Geophys. Res. Lett., 33, L07104, doi:10.1029/2005GL025522.

Desch, M. D., and M. L. Kaiser (1981), Voyager measurement of the rotation period of Saturn's magnetic field, Geophys. Res. Lett., 8(3), 253-256, doi:10.1029/GL008i003p00253.

Dougherty, M. K., et al. (2004), The Cassini magnetic field investigation, Space Sci. Rev., 114, 331-383.

Dougherty, M. K., et al. (2005), Cassini magnetometer observations during Saturn orbit insertion, Science, 307, 1266-1270, doi:10.1126/ science. 1106098 .

Espinosa, S. A., and M. K. Dougherty (2000), Periodic perturbations in Saturn's magnetic field, Geophys. Res. Lett., 27(17), 2785-2788, doi:10.1029/2000GL000048.

Espinosa, S. A., D. J. Southwood, and M. K. Dougherty (2003a), Reanalysis of Saturn's magnetospheric field data in view of spin periodic perturbations, J. Geophys. Res., 108(A2), 1085, doi:10.1029/2001JA005083.

Espinosa, S. A., D. J. Southwood, and M. K. Dougherty (2003b), How can Saturn impose its rotation period in a noncorotating magnetosphere?, J. Geophys. Res., 108(A2), 1086, doi:10.1029/2001JA005084.

Fischler, M. A., and R. C. Bolles (1981), Random sample consensus: A paradigm for model fitting with applications to image analysis and automated cartography, Comm. ACM, 24(6), 381-395, doi:10.1145/ 358669.358692

Galopeau, P., A. Ortega-Molina, and P. Zarka (1991), Evidence of Saturn's magnetic field anomaly from Saturnian kilometric radiation high-frequency limit, J. Geophys. Res., 96(A8), 14,129-14,140, doi:10.1029/91JA00696.

Galopeau, P. H. M., and A. Lecacheux (2000), Variations of Saturn's radio rotation period measured at kilometer wavelengths, J. Geophys. Res., 105 (A6), 13,089-13,101, doi:10.1029/1999JA005089.

Galopeau, P. H. M., P. Zarka, and D. Le Quéau (1995), Source location of Saturn's kilometric radiation: The Kelvin-Helmholtz instability hypothesis, J. Geophys. Res., 100(E12), 26,397-26,410, doi:10.1029/95JE02132.

Giampieri, G., M. K. Dougherty, E. J. Smith, and C. T. Russell (2006), A regular rotation period for Saturn's magnetic field that may track its internal rotation, Nature, 441, 62-64, doi:10.1038/nature04750.

Goldreich, P., and A. J. Farmer (2007), Spontaneous axisymmetry breaking of the external magnetic field at Saturn, J. Geophys. Res., 112, A05225, doi:10.1029/2006JA012163.

Gurnett, D. A., et al. (2004), The Cassini radio and plasma wave investigation, Space Sci. Rev., 114, 395-463.

Gurnett, D. A., et al. (2005), Radio and plasma wave observations at Saturn from Cassini's approach and first orbit, Science, 307, 1255-1259, doi:10.1126/science. 1105356 .

Gurnett, D. A., A. M. Persoon, W. S. Kurth, J. B. Groene, T. F. Averkamp, M. K. Dougherty, and D. J. Southwood (2007), The variable rotation period of the inner region of Saturn's plasma disk, Science, 316 , 442-445, doi: 10.1126 /science. 1138562 .

Gurnett, D. A., A. Lecacheux, W. S. Kurth, A. M. Persoon, J. B. Groene, L. Lamy, P. Zarka, and J. F. Carbary (2009a), Discovery of a northsouth asymmetry in Saturn's radio rotation period, Geophys. Res. Lett., 36, L16102, doi:10.1029/2009GL039621.

Gurnett, D. A., A. M. Persoon, J. B. Groene, A. J. Kopf, G. B. Hospodarsky, and W. S. Kurth (2009b), A north-south difference in the rotation rate of auroral hiss at Saturn: Comparison to Saturn's kilometric radio emission, Geophys. Res. Lett., 36, L21108, doi:10.1029/2009GL040774.

Horne, J. H., and S. L. Baliunas (1986), A prescription for period analysis of unevenly sampled time series, Astrophys. J., 302, 757-763, doi:10.1086/ 164037.

Kaiser, M. L., M. D. Desch, J. W. Warwick, and J. B. Pearce (1980), Voyager detection of nonthermal radio emission from Saturn, Science, 209 , 1238-1240, doi:10.1126/science.209.4462.1238.

Kellett, S., C. S. Arridge, E. J. Bunce, A. J. Coates, S. W. H. Cowley, M. K. Dougherty, A. M. Persoon, and N. Sergis (2010), Nature of the ring current in Saturn's dayside magnetosphere, J. Geophys. Res., 115 A08201, doi:10.1029/2009JA015146.

Khurana, K. K., D. G. Mitchell, C. S. Arridge, M. K. Dougherty, C. T. Russell, C. Paranicas, N. Krupp, and A. J. Coates (2009), Sources of rotational signals in Saturn's magnetosphere, J. Geophys. Res., 114, A02211, doi:10.1029/2008JA013312.

Krupp, N., et al. (2005), The Saturnian plasma sheet as revealed by energetic particle measurements, Geophys. Res. Lett., 32, L20S03, doi:10.1029/2005GL022829.

Kurth, W. S., A. Lecacheux, T. F. Averkamp, J. B. Groene, and D. A. Gurnett (2007), A Saturnian longitude system based on a variable kilometric radiation period, Geophys. Res. Lett., 34, L02201, doi:10.1029/2006GL028336.

Kurth, W. S., T. F. Averkamp, D. A. Gurnett, J. B. Groene, and A. Lecacheux (2008), An update to a Saturnian longitude system based on kilometric radio emissions, J. Geophys. Res., 113, A05222, doi:10.1029/2007JA012861.

Lamy, L., P. Zarka, B. Cecconi, R. Prangé, W. S. Kurth, and D. A. Gurnett (2008a), Saturn kilometric radiation: Average and statistical properties, J. Geophys. Res., 113, A07201, doi:10.1029/2007JA012900.

Lamy, L., P. Zarka, B. Cecconi, S. Hess, and R. Prangé (2008b), Modelling of Saturn kilometric radiation arcs and equatorial shadow zone, J. Geophys. Res., 113, A10213, doi:10.1029/2008JA013464.

Lamy, L., B. Cecconi, R. Prangé, P. Zarka, J. D. Nichols, and J. T. Clarke (2009), An auroral oval at the footprint of Saturn's kilometric radio sources, colocated with the UV aurorae, J. Geophys. Res., 114, A10212, doi:10.1029/2009JA014401.

Lamy, L., et al. (2010), Properties of Saturn kilometric radiation measured within its source region, Geophys. Res. Lett., 37, L12104, doi:10.1029/ 2010GL043415

Lecacheux, A., and F. Genova (1983), Source localization of Saturn kilometric radio emission, J. Geophys. Res., 88(A11), 8993-8998, doi:10.1029/JA088iA11p08993.

Lomb, N. R. (1976), Least-squares frequency analysis of unequally spaced data, Astrophys. Space Sci., 39, 447-462, doi:10.1007/BF00648343.

Nichols, J. D., J. T. Clarke, S. W. H. Cowley, J. Duval, A. J. Farmer, J.-C. Gérard, D. Grodent, and S. Wannawichian (2008), Oscillation of Saturn's southern auroral oval, J. Geophys. Res., 113, A11205, doi:10.1029/2008JA013444.

Nichols, J. D., B. Cecconi, J. T. Clarke, S. W. H. Cowley, J.-C. Gérard, A. Grocott, D. Grodent, L. Lamy, and P. Zarka (2010), Variation of Saturn's UV aurora with SKR phase, Geophys. Res. Lett., 37, L15102, doi:10.1029/2010GL044057.

Provan, G., D. J. Andrews, C. S. Arridge, S. W. H. Cowley, S. E. Milan, M. K. Dougherty, and D. M. Wright (2009a), Polarization and phase of planetary period oscillations on high latitude field lines in Saturn's magnetosphere, J. Geophys. Res., 114, A02225, doi:10.1029/ 2008JA013782.

Provan, G., S. W. H. Cowley, and J. D. Nichols (2009b), Phase relation of oscillations near the planetary period of Saturn's auroral oval and the equatorial magnetospheric magnetic field, J. Geophys. Res., 114 A04205, doi:10.1029/2008JA013988.

Scargle, J. D. (1982), Studies in astronomical time series analysis: II. Statistical aspects of spectral analysis of unevenly spaced data, Astrophys. $J ., 263,835-853$, doi:10.1086/160554

Sergis, N., et al. (2010), Particle pressure, inertial force and ring current density profiles in the magnetosphere of Saturn, based on Cassini measurements, Geophys. Res. Lett., 37, L02102, doi:10.1029/ 2009GL041920.

Southwood, D. J., and M. G. Kivelson (2007), Saturn magnetospheric dynamics: Elucidation of a camshaft model, J. Geophys. Res., 112, A12222, doi:10.1029/2007JA012254

Southwood, D. J., and M. G. Kivelson (2009), The source of Saturn's periodic radio emission, J. Geophys. Res., 114, A09201, doi:10.1029/ 2008JA013800.

Talboys, D. L., C. S. Arridge, E. J. Bunce, A. J. Coates, S. W. H. Cowley, and M. K. Dougherty (2009a), Characterization of auroral current systems in Saturn's magnetosphere: High-latitude Cassini observations, J. Geophys. Res., 114, A06220, doi:10.1029/2008JA013846.

Talboys, D. L., C. S. Arridge, E. J. Bunce, A. J. Coates, S. W. H. Cowley, M. K. Dougherty, and K. K. Khurana (2009b), Signatures of fieldaligned currents in Saturn's nightside magnetosphere, Geophys. Res. Lett., 36, L19107, doi:10.1029/2009GL039867.

Warwick, J., et al. (1981), Planetary radio astronomy observations from Voyager-1 near Saturn, Science, 212(4491), 239-243.

$\mathrm{Wu}, \mathrm{C}$. S., and L. C. Lee (1979), A theory of the terrestrial kilometric radiation, Astrophys. J., 230, 621-626, doi:10.1086/157120.

Young, D., et al. (2004), Cassini Plasma Spectrometer investigation, Space Sci. Rev., 114(1-4), 1-112. 
Zarka, P. (1998), Auroral radio emissions at the outer planets: Observations and theories, J. Geophys. Res., 103(E9), 20,159-20,194, doi:10.1029/ 98JE01323

Zarka, P., L. Lamy, B. Cecconi, R. Prangé, and H. O. Rucker (2007), Modulation of Saturn's radio clock by solar wind speed, Nature, $450,265-267$.

D. J. Andrews, S. W. H. Cowley, and G. Provan, Department of Physics and Astronomy, University of Leicester, Leicester, LE1 7RH, UK. (dja13@ion.le.ac.uk)
A. J. Coates, Mullard Space Science Laboratory, University College London, Dorking, RH5 6NT, UK.

M. K. Dougherty, Blackett Laboratory, Imperial College London, London, SW7 2AZ, UK

L. Lamy and P. Zarka, LESIA, Observatoire de Paris, CNRS, UPMC, Université Paris Diderot, Meudon, France. 